\title{
HAVE MUSICIANS' MUSCULOSKELETAL SYMPTOMS BEEN THOROUGHLY ADDRESSED? A SYSTEMATIC MAPPING REVIEW
}

JESSICA STANHOPE ${ }^{1}$, REBECCA TOOHER ${ }^{1}$, DINO PISANIELLO ${ }^{1}$, and PHILIP WEINSTEIN ${ }^{2}$

The University of Adelaide, Adelaide, Australia

${ }^{1}$ School of Public Health

${ }^{2}$ School of Biological Sciences

\begin{abstract}
The authors aimed to characterize the current evidence base regarding musicians' musculoskeletal symptoms (MSS), in order to identify gaps for future research. A systematic search was conducted to identify peer-review studies published in English in 2007-2016 that investigated musicians' MSS. Narrative reviews, case reports, protocols, and questionnaire or program development papers were excluded. Data were synthesized descriptively in order to identify gaps in the current evidence base. Five systematic reviews and 153 primary studies (133 unique) were included in this review. The majority $(71 \%)$ of studies investigated professional musicians and/or university music students, with orchestral musicians being the most commonly investigated group. The majority of studies investigated the extent of the problem (68\%) and/or associated factors (54\%). Eight studies compared the prevalence of MSS outcomes with other populations. A range of risk factors were investigated; however, few studies used longitudinal designs. A total of 16 intervention studies were identified ( 3 clinical, 13 public health), with 12 investigating education or exercise programs. There is a need for research into musicians beyond classical university music students and professional orchestral musicians, and these musical sub-groups should be compared to determine the most at risk groups of musicians. Studies looking at potential risk factors should move towards longitudinal designs so that the temporal relationship of these factors and MSS could be established, where cross-sectional designs have indicated that an association exists. Intervention studies should be based upon the risk factors identified, and extend beyond education and exercise programs. Int J Occup Med Environ Health. 2019;32(3):291-331
\end{abstract}

Key words:

pain, musculoskeletal pain, literature review, music, musculoskeletal diseases, occupational diseases

\section{INTRODUCTION}

Musculoskeletal disorders (MSDs) impact significantly on the general population, and are the leading cause of years lived with disability globally [1]. The prevalence of musculoskeletal symptoms (MSS) is high among workers, with the 12 month prevalence as $92 \%$ [2]. However, there are a number of under-investigated groups, including subgroups of the agricultural [3-5], science [6], health [7,8], and catering [9] industries, as well as musicians [10].
Ramazzini was the first to investigate the health issues experienced by musicians, including a chapter on musicians in his De Morbis Artificum Diatriba, published in 1700 [11]. Despite the acknowledgement of musicians' health issues over 300 years ago, it was not until the late 1980s, with the initial publication of Medical Problems of Performing Artists in 1986 [12], and the inception of the Performing Arts Medicine Association in 1989 [13], that musicians' health became an established field of research and practice.

Received: March 20, 2018. Accepted: January 10, 2019.

Corresponding author: Jessica Stanhope, The University of Adelaide, School of Public Health, Adelaide Health and Medical Sciences Building, Crn George St \& North Tce, Adelaide 5000, Australia (e-mail: jessica.stanhope@adelaide.edu.au). 
Musicians experience a range of health problems, including neurological [14,15], musculoskeletal [16-19], respiratory [20,21], auditory [22,23], dermatological [24-26], and psychological conditions [27], which may be the result of or impair musical activity. Musculoskeletal symptoms are one of the most common health complaints experienced by musicians, with the 12 month prevalence of MSS in professional musicians and university music students reported as 86$89 \%$ [19]. Musculoskeletal symptoms may result in a range of participation restrictions and activity limitations [28-30], and in some cases they have led musicians to change their careers [31,32], or to stop playing permanently [33].

The broad nature of MSS, the diversity of musicians as a population, and the wide range of potential risk factors and interventions for musicians' MSS mean that despite the large number of studies conducted into musicians' MSS over the last 30 years, it is likely that there are still under-investigated sub-populations and unanswered research questions. There is little current guidance regarding gaps in the research into musicians' MSS, which may lead to unnecessary replication of studies, or sub-populations of musicians (e.g., opera musicians, military band musicians) remaining under-investigated. The present research, therefore, uses a systematic mapping review approach to provide a comprehensive analysis of the current evidence base.

This systematic mapping review aims to characterize the recent research (published in 2007-2016) regarding musicians' MSS, and to identify gaps in the recent research evidence base. Specifically, the authors were interested in the subpopulations investigated, the types of studies conducted, and the types of outcomes analyzed, as well as the associated factors or mechanisms for MSS, and interventions that had been investigated. In addition, other research topics relating to musicians' MSS were described.

\section{METHODS}

A systematic mapping review is an appropriate method for identifying gaps in the literature and may guide deci- sions regarding future research [34]. Systematic mapping reviews utilize transparent and explicit methods to search for literature, with synthesis typically being tabular and/ or graphical [34]. There is no formal quality assessment in these types of reviews, although study designs may be reported [34]. These reviews characterize the literature according to key features, which may include the population, setting and/or theoretical perspective [34].

\section{Database search strategy}

Seven databases (Ovid Medline, Ovid Embase, EbscoHost Health Source: Nursing and Academic Edition, EbscoHost Cumulative Index to Nursing and Allied Health Literature, EbscoHost Music Index, Web of Science Core Collection, and Cochrane Library) were searched in January 2017 using search terms relating to musicians and MSS (Table 1). Terms were searched in the title and keyword fields, and subject headings (e.g., MeSH and Emtree) were searched as applicable. In addition to specific MSS terms, broader search terms, e.g., health* and medical*, were also included in order to capture studies that might report MSS specifically within the full text. Where possible searches were limited to the English language, and pubication dates to 2007-2016. The search strategy was developed through a review of previous search strategies regarding musicians' MSS [10,16-19,35,36], scoping searches, and the assistance of an academic librarian. All studies identified in the database searches were exported into EndNote X7.

\section{Manual search of Medical Problems of Performing Artists}

The titles of articles published in Medical Problems of Performing Artists [12] in 2007-2016 were screened, as were the abstracts sections of this journal. Titles were deemed potentially relevant if they mentioned musicians or performing artists, as well as MSS, health or medical conditions. Potentially relevant studies were added to the list of studies identified through the database search. 
Table 1. Search terms used in the search for the studies on musicians' musculoskeletal symptoms published in 2007-2016

\begin{tabular}{|c|c|}
\hline Search field & Musician terms \\
\hline Title or keyword & $\begin{array}{l}\text { musician* OR "music-related" OR "music related" OR } \\
\text { conservatory OR conservatories OR conservatorium* OR } \\
\text { conservatoire* OR woodwind* OR flute OR flutes OR flautist* } \\
\text { OR flutist* OR clarinet* OR sax OR saxes OR saxophon* } \\
\text { OR *bassoon* OR oboe* OR oboist* OR "double reed*" OR } \\
\text { "Double-reed*" OR trumpet* OR trombon* OR tuba OR } \\
\text { tubas OR tubist* OR bugle* OR cornet* OR euphonium* OR } \\
\text { violin* OR violinist* OR viola OR violas OR violist* OR *cello } \\
\text { OR *cellos OR *cellist* OR guitar* OR fiddle* OR banjo* OR } \\
\text { baritone* OR piano* OR pianist* OR timpan* OR hornist* } \\
\text { OR bassist* OR bagpipe* OR drummer* OR percussionist* } \\
\text { OR harpist* OR harp OR harps OR harpsichord* OR organist* } \\
\text { OR "church organ*" OR "pipe organ*" OR keyboardist* OR } \\
\text { instrumentalist* OR vocalist* OR sing OR singer* OR singing } \\
\text { OR choir* OR orchestra OR orchestras OR "playing-related" OR } \\
\text { "performance-related" OR "playing related" OR "performance } \\
\text { related" OR musical* OR (music NEAR/3 (major* OR stud* } \\
\text { OR teach* OR tuition OR training OR educat* OR school* OR } \\
\text { perform* OR rehear* OR play* OR practi* OR concert* OR } \\
\text { band* OR ensemble* or instrument*)) OR ((*wind* OR *reed* } \\
\text { OR brass OR string* OR horn* OR bass* OR recorder* OR } \\
\text { pipe OR pipes OR piper OR pipers OR drum* OR percussion* } \\
\text { OR organ OR organs OR keyboard* OR vocal* OR voice) } \\
\text { NEAR/3 (major* OR stud* OR teach* OR tuition OR training } \\
\text { OR educat* OR school* OR perform* OR rehear* OR play* OR } \\
\text { practi* OR concert* OR band* OR ensemble* OR instrument* } \\
\text { OR music* OR corp OR corps)) OR "instrumental music*” OR } \\
\text { ((band* OR ensemble*) NEAR/3 (music* OR stage OR big OR } \\
\text { folk OR country OR brass OR wind OR string OR percussion } \\
\text { OR jazz OR baroque OR Dixieland OR traditional OR Irish OR } \\
\text { march* OR military OR army OR defence OR navy OR force } \\
\text { OR member*)) OR "marching art*" OR "performing art*” OR } \\
\text { (conductor* NEAR/3 (music* OR band* OR orchestra* OR } \\
\text { ensemble*)) OR "musical director*” OR "drum major*" OR } \\
\text { opera OR operas OR operatic }\end{array}$ \\
\hline
\end{tabular}

\section{$\mathrm{MeSH}$}

Emtree Musician

CINAHL subject heading

Health Source subject headings

\section{Musculoskeletal health terms}

Medical* OR health* OR *musculo*

OR *skeletal* OR muscle* OR joint*

OR arthr* OR tendon* OR tendin*

OR ligament* OR strain* OR sprain*

OR injur* OR fracture* OR pain*

OR *ache OR aching OR weakness

OR tingl* OR "pins and needles" OR

numb* OR *mobilit* OR *flexib*

OR stiff* OR "range of motion" OR

"range-of-motion" OR tight* OR

swell* OR *edema OR *ordinat* OR

disorder* OR condition* OR symptom*

OR "soft tissue*" OR dysfunction* OR

nerve* OR neuro* OR orthop*
Musculoskeletal diseases OR pain OR "wounds and injuries"

Musculoskeletal disease OR pain OR injury

Musculoskeletal diseases OR pain OR "wounds and injuries"

Musculoskeletal system - diseases OR pain or "wounds \& injuries"

* Truncation.

NEAR/3 - up to 3 words between.

All subject heading terms were explored where available. 


\section{Study inclusion/exclusion}

The process of study inclusion/exclusion is outlined in Figure 1 . Where there was any uncertainty regarding the inclusion or exclusion of studies based on the title and abstract, these were retained. The full texts of the remaining studies were obtained and screened against the same criteria. If there was any uncertainty regarding study inclusion or exclusion, the views of other authors were sought.

\section{Reference and citation list searching}

The reference and citation lists of included studies, and the relevant narrative reviews, case reports, editorials and correspondence were screened to identify potentially relevant studies. Citation lists were obtained from Google

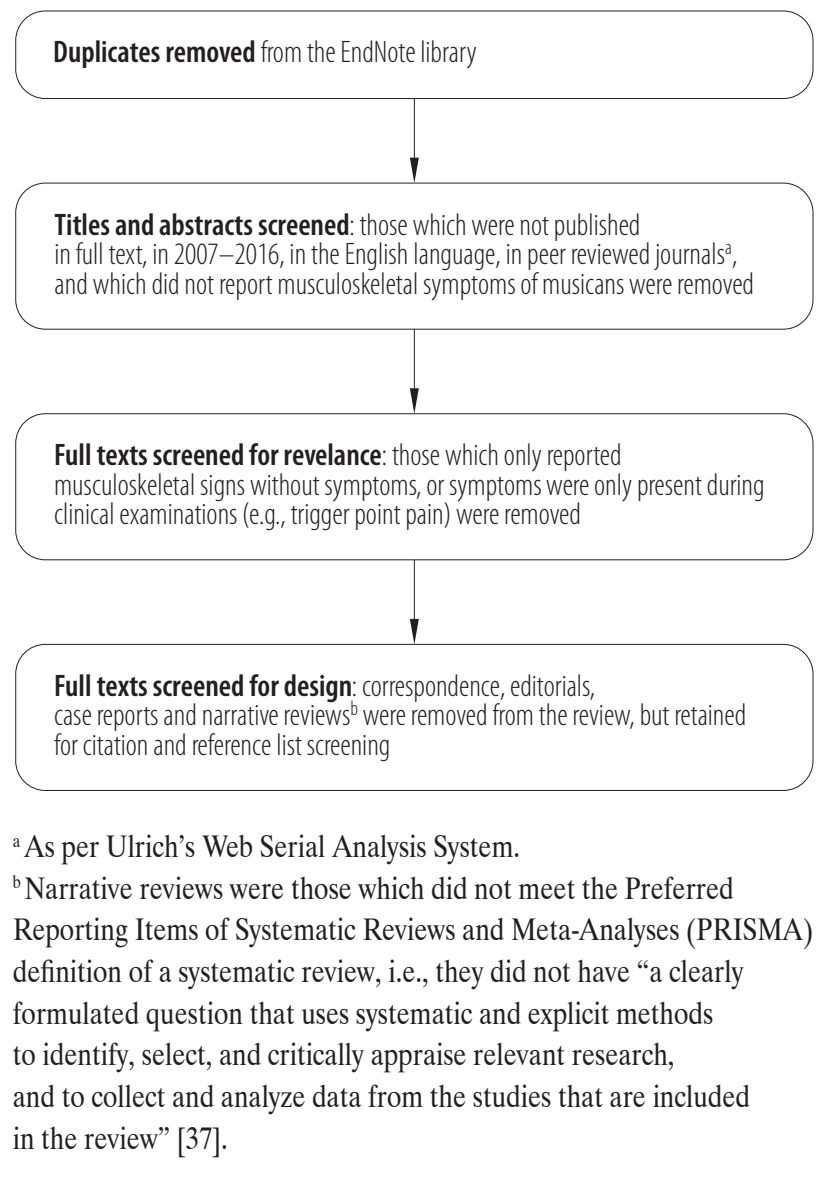

Figure 1. Inclusion/exclusion criteria in the study on musicians' musculoskeletal symptoms (MSS)
Scholar and Web of Science (January 2017). Potentially relevant studies were then screened against the inclusion/ exclusion criteria. This process continued until no additional studies were identified.

\section{Data extraction and analysis}

Data were extracted manually by the first author, with each article extraction checked independently by another author. Data were extracted into a purpose-built table. Studies were classified as the extent of the problem (e.g., prevalence (this included studies where the prevalence could be calculated, i.e., the number of affected participants, and the sample size was reported), incidence, severity), associated factors and mechanisms (associations had to be reported statistically), interventions, and "other" (for studies that did not fit within the other categories), as based upon van Mechelan et al.'s [37] model of injury prevention research.

Extracted data included:

- the year of data collection and publication,

- the country in which the study was conducted (if this was not reported, the country of ethics approval, funding and/or author affiliations was used),

- the study design,

- the population level:

- professional ("professional” status was applied only when the study reported the population as being professional),

- professional and university music students,

- university music students,

- adults (all $\geq 18$ years, or the mean minus the standard deviation was $\geq 18$ years),

- children (did not meet the adult criteria),

- mixed (adults and children reported together),

- musical genre (e.g., classical, or jazz),

- ensemble type,

- type of an extent sudy (e.g., prevalence, incidence),

- mechanisms or associated factors, 
- interventions,

- MSS outcomes investigated:

- MSS in general (including specific diagnoses and where "playing-related" was not defined, or inconsistent terminology was used),

- MSS with perceived risk or aggravating factors,

- MSS consequences (e.g., time off from playing, treatment).

In keeping with the systematic mapping review design, data were presented in tabular and graphical forms [34], supported with narrative descriptions.

\section{RESULTS}

A total of 158 articles were included (Figure 2). Some studies utilized the same datasets as others $[27,28,32,38-$ 66], resulting in 138 unique studies.

\section{Systematic reviews}

Five systematic reviews were included [10,17-19,35], 4 of which were published in 2015-2016. Professional musicians and university music students $[10,19,35]$, flautists of any age and level [17], and instrumentalists of any age and level [18] were investigated. Three also systematic reviews investigated the extent of the problem; namely, prevalence [17-19] and episodic incidence [17]. Three reported MSS in general, and the consequences of symptoms, with all reporting that the included studies did not define "playing-related" adequately [17-19]. Silva et al. [18] also reported on symptoms caused by playing the piano and preventing piano being played (classified as mixed age and level).

Four systematic reviews investigated factors associated with MSS outcomes in musicians [10,18,19,35]. Two focused on risk factors [10,35], and 2 focused on prevalence, but also investigated the differences in prevalence by gender [18,19], age [19], instrument group [19], and occupational group (e.g., teachers, orchestral) [19]. These systematic reviews investigated professional musicians and university music students [10,19], professional instrumentalists and university instrumental students [35], and instrumentalists of mixed ages/levels [18]. Baadjou et al. [35] excluded musicians in marching bands, and also appeared to have excluded instrumental music teachers; however, the reason for that exclusion was not given. In addition to investigating factors associated with MSS outcomes, $\mathrm{Wu}[10]$ also reported the percentage of participants agreeing with the perceived contributing factors; however, it was unclear whether these were specifically for their own symptoms or more general perceptions.

\section{Primary studies}

Of the 133 unique primary studies, $68 \%$ investigated the extent or severity of MSS, $54 \%$ associated factors or mechanisms, $12 \%$ interventions, and $32 \%$ other topics. There was a steady rise in the number of studies published over the last 10 years, particularly for the extent/severity and associated factors/mechanism studies (Figure 3). Values reported henceforth refer to the number or percentage of unique primary studies.

The majority of primary studies $(85 \%)$ were from highincome countries (as per the United Nations [67]). This was the case across all types of studies, with $82 \%$ of extent/severity studies, $85 \%$ of associated factors/mechanisms, $100 \%$ of intervention studies, and $80 \%$ of other study types coming from high-income countries. Overall, most unique studies were from Europe (38\%) or North America (29\%), with these continents dominating most study types.

\section{Extent/severity studies}

The majority (71\%) of studies investigated professional musicians and/or university students, with professional and/or university student orchestral or classical musicians being the most commonly investigated (32\%) (Table 2). While the players of a range of non-Western instruments were investigated, those playing Western instruments were most commonly investigated. Regarding specific in- 
Studies from the database search $(N=6575)$

- Cumulative Index of Nursing and Allied Health Literature ( $N=517)$

- Cochrane $(N=767)$

- Embase $(\mathrm{N}=2035)$

- Health Source $(\mathrm{N}=117)$

- Medline ( $N=722)$

- Music Index ( $N=780)$

- Web of Science $(N=1637)$
Studies from Medical Problems of Performing Artists [12] ( $N=131$

- table of contents $(N=116)$

- abstracts $(N=15)$

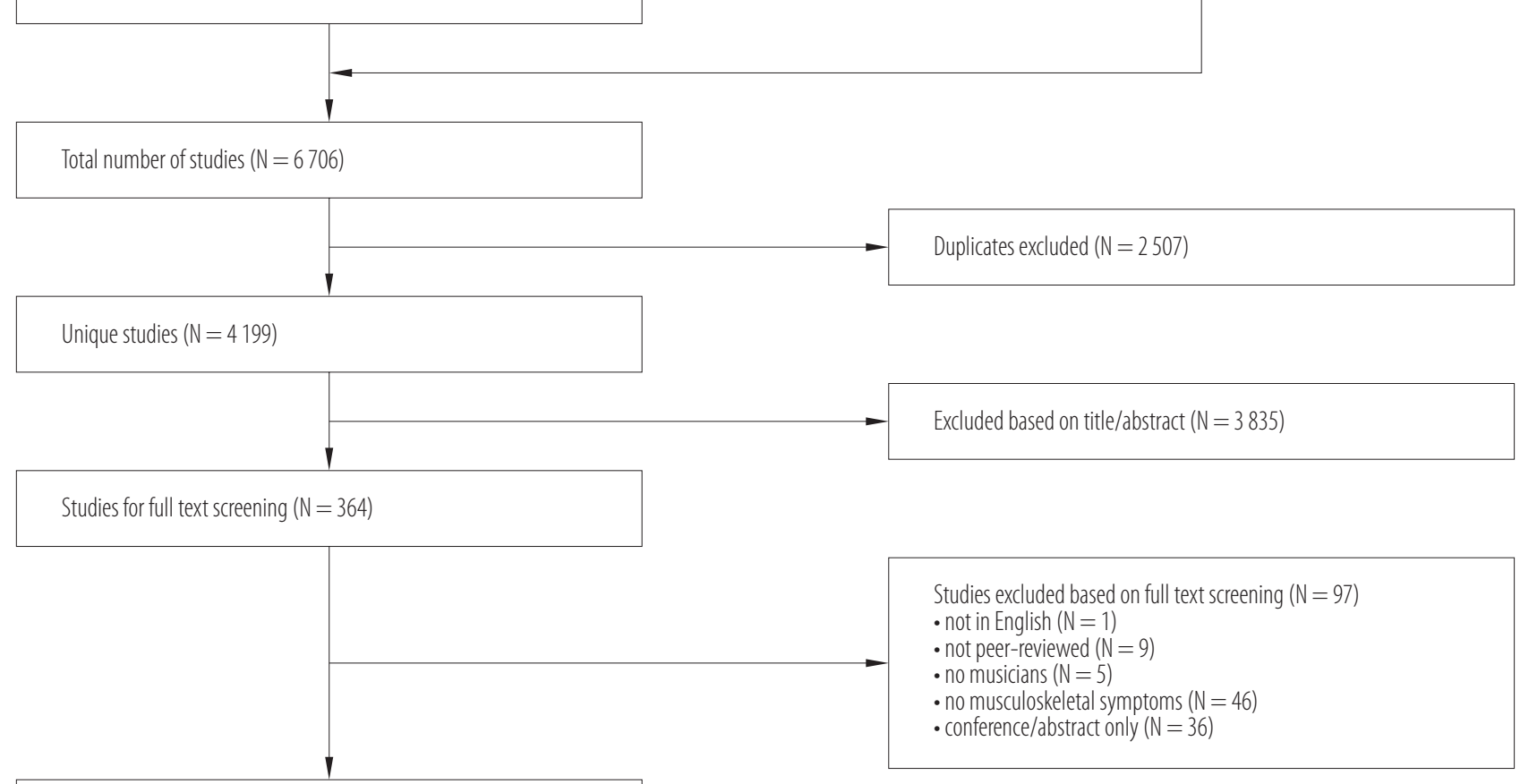

Relevant studies $(\mathrm{N}=267)$

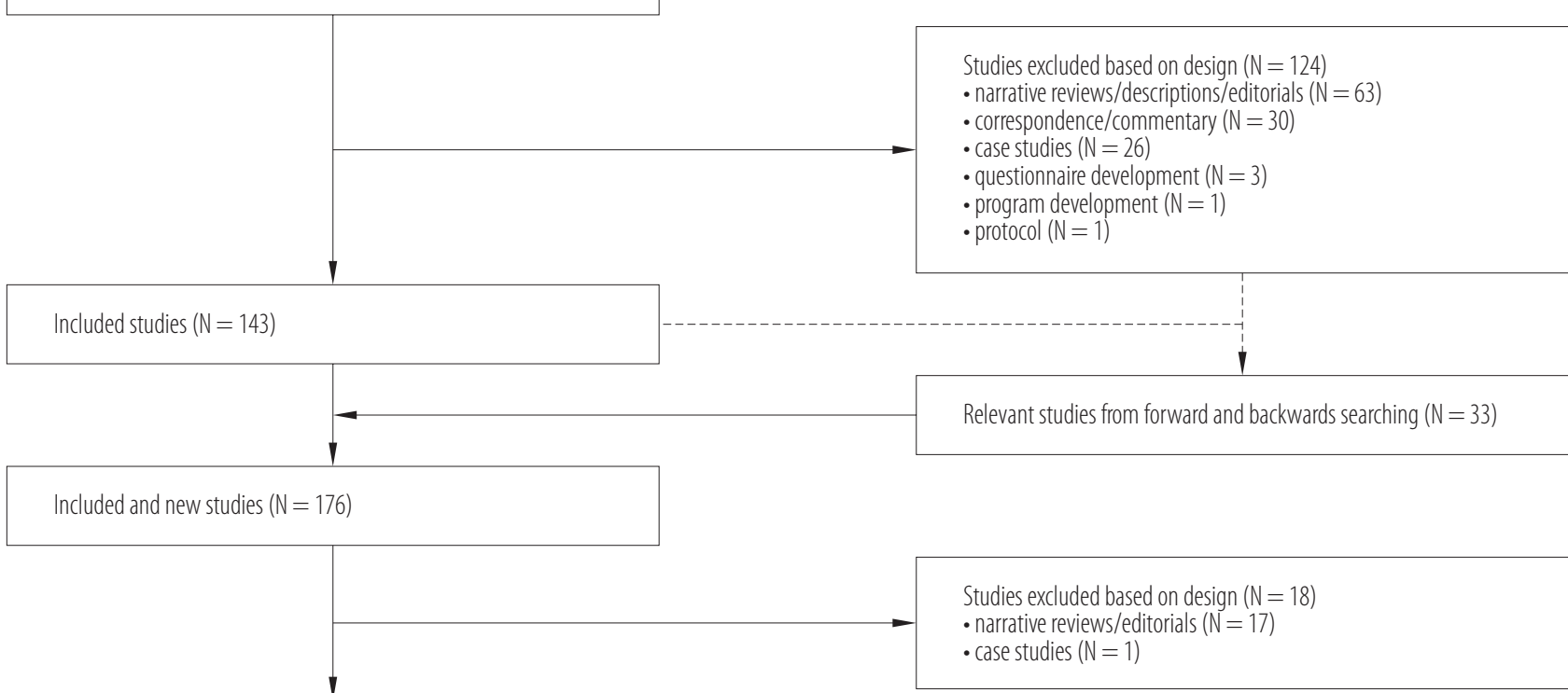

Total included studies ( $N=158$

Figure 2. Flowchart of the inclusion/exclusion criteria in the study on musicians' musculoskeletal symptoms 
struments, the most commonly investigated were flautists $(10 \%)$, trombonists $(10 \%)$, and pianists (14\%) (Table 3).

When instruments were grouped, they tended to be within traditional instrument groupings (e.g., woodwind, brass, strings, percussion); however, there were some exceptions. Some appeared to be collections of instruments where there were small numbers of participants (e.g., keyboards, or harps in orchestras) $[46,54,93]$, while others grouped instruments according to their biomechanical exposures; namely, symmetrical or asymmetrical playing postures [93]; whether their musical activity (e.g., instrument or singing) was thought by the researchers to place

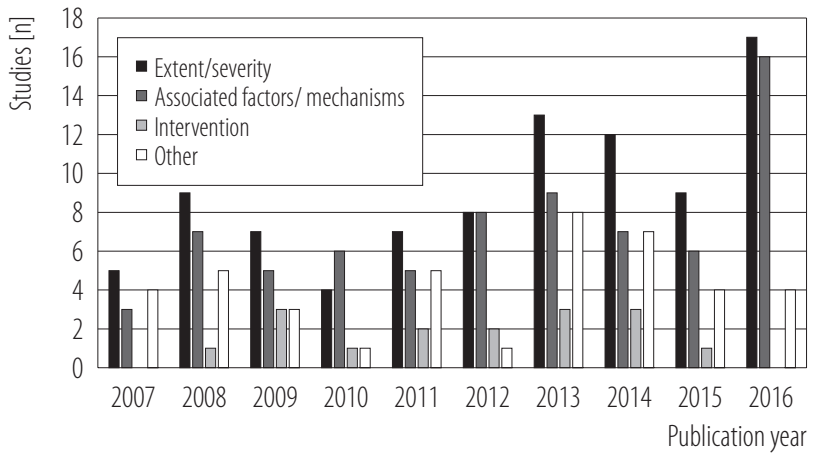

Where there were duplicate reports of a study, the oldest publication was counted.

Figure 3. Publications on musicians' musculoskeletal symptoms by year of publication

Table 2. Type of musicians in the studies on musicians' musculoskeletal symptoms published in 2007-2016

\begin{tabular}{|c|c|c|c|c|}
\hline \multirow[b]{2}{*}{ Musician type } & \multicolumn{4}{|c|}{ Reference } \\
\hline & extent/severity & $\begin{array}{l}\text { associated factors/ } \\
\text { mechanisms }\end{array}$ & intervention & other \\
\hline Professional & $\begin{array}{l}27-30,45,46,60,64-66 \\
68-95\end{array}$ & $\begin{array}{l}\text { 27-30, 45, 46, 60, 64, 65, } \\
71,74,76,79-88,90-93 \text {, } \\
95-97\end{array}$ & $\begin{array}{l}58,59,68,90, \\
98-100\end{array}$ & $\begin{array}{l}29,30,32,51,52,61-63, \\
68,73,85,92,94, \\
101-103,104\end{array}$ \\
\hline orchestral & $\begin{array}{l}27-30,45,46,60,64-66 \\
68,71,72,74,76,78-81 \\
83,87,91,92,94,95\end{array}$ & $\begin{array}{l}\text { 27-30, 45, 46, 60, 64, 65, } \\
71,74,76,79-81,83,87 \\
91,92,95\end{array}$ & $\begin{array}{l}58,59,68 \\
98-100\end{array}$ & $\begin{array}{l}29,30,61-63,68,92 \\
94,103\end{array}$ \\
\hline classical & $45,78,80,86$ & $45,80,86,97$ & & 32,52 \\
\hline symphony or philharmonic & $\begin{array}{l}28,30,60,68,74,76,79 \\
81,94,95\end{array}$ & $\begin{array}{l}28,30,60,74,76,79 \\
81,95\end{array}$ & 68,100 & $30,68,94$ \\
\hline state or opera & 27 & 27 & & \\
\hline symphony or opera & 29 & 29 & & 29 \\
\hline symphony or pit & 65,66 & 65 & & \\
\hline stage and pit & 64 & & & \\
\hline stage & 64 & & & \\
\hline pit & 64 & & & \\
\hline military band & $84,85,89,90$ & $84,85,90$ & 90 & 85 \\
\hline blues & 90 & & & \\
\hline ceremonial & 90 & & & \\
\hline chorus & 90 & & & \\
\hline concert & 90 & & & \\
\hline Cuban band & 73 & & & 73 \\
\hline opera singers & & & & 104 \\
\hline classical choristers & 75 & & & \\
\hline
\end{tabular}


Table 2. Type of musicians in the studies on musicians' musculoskeletal symptoms published in 2007-2016 - cont.

\begin{tabular}{|c|c|c|c|c|}
\hline \multirow[b]{2}{*}{ Musician type } & \multicolumn{4}{|c|}{ Reference } \\
\hline & extent/severity & $\begin{array}{l}\text { associated factors/ } \\
\text { mechanisms }\end{array}$ & intervention & other \\
\hline \multicolumn{5}{|l|}{ Professional - cont. } \\
\hline teachers & $69,82,93$ & 82,93 & & 102 \\
\hline jazz & & & & 102 \\
\hline Professional or university students & $91,105-109$ & $91,106-108,110,111$ & 112,113 & 105,114 \\
\hline University students & $53-55,91,102,115-139$ & $\begin{array}{l}\text { 53, 54, 91, 115, 116, 119, } \\
\text { 122, 125-127, 129-131, } \\
133-142\end{array}$ & $143-148$ & $\begin{array}{l}55,102,121,124,125 \\
128,129,136,137,139 \\
149-152\end{array}$ \\
\hline orchestral & 116 & 116 & & \\
\hline symphony orchestra & 134 & 134 & & \\
\hline marching band & $117,130,132$ & 130 & & \\
\hline classical & $53-55,127,128,136$ & $53,54,136$ & & $55,128,136,150$ \\
\hline jazz & 102,118 & & & 102 \\
\hline rhythmic & 127 & & & \\
\hline musical theatre & 139 & 139 & & 139 \\
\hline Adults & $49,50,54,77,153-161$ & $\begin{array}{l}49,50,153-157 \\
159-160,162-164\end{array}$ & & $56,57,158,166-169$ \\
\hline orchestral & 153 & 153 & & \\
\hline band & 158 & & & 158 \\
\hline drum and bugle corp & 159 & 159 & & \\
\hline Korean traditional & 49,50 & 49,50 & & \\
\hline Irish traditional & & & & 56,57 \\
\hline Children & $\begin{array}{l}\text { 40-44, 47, 48, 136, } \\
170-177\end{array}$ & $\begin{array}{l}40-44,47,48,136,170 \\
172-174,178\end{array}$ & 179,180 & 136,177 \\
\hline orchestral & 172 & 172 & 179,180 & \\
\hline classical & 136 & 136 & & 136 \\
\hline marching band & 174 & 174 & & \\
\hline Mixed & $38,39,69,136,181-185$ & $38,69,136,182,183$ & & $39,136,183,184,186$ \\
\hline classical & 136 & 136 & & 136 \\
\hline
\end{tabular}

This classification refers to the target population that was not necessarily the same as those from whom data were collected (e.g., Rickert et al. [61-63,151], Ackermann and Driscoll [175], Ajidahun and Phillips [186], and McKechnie and Jacobs [177]).

them at increased risk of orofacial pain [74], and whether the arms were elevated $\geq 40^{\circ}$ for playing [83,182]. Regarding the latter, Nyman et al. [83] further divided groups by playing time, resulting in 4 groupings that included "tra- ditional groupings" of violin and viola, as well as cello and double bass.

In addition to instrumentalists, drum majors [132], composers [125], vocalists [73,75,77,125,127,135,139], and vo- 
Table 3. Instruments and instrument groups investigated

\begin{tabular}{|c|c|c|c|c|}
\hline \multirow[b]{2}{*}{ Instrument type } & \multicolumn{4}{|c|}{ Reference } \\
\hline & extent/severity & $\begin{array}{l}\text { associated factors/ } \\
\text { mechanisms }\end{array}$ & $\begin{array}{l}\text { interven- } \\
\text { tion }\end{array}$ & other \\
\hline Wind & $47,79,84,93,116,127,170$ & $79,84,116$ & & \\
\hline symmetrical wind & & & & 166 \\
\hline woodwind & $\begin{array}{l}30,41,44-46,54,60,65,66,76,86,119,122 \\
125,128-131,135,138,158\end{array}$ & 122,130 & & $68,114,128$ \\
\hline non-reed/aerophone & 129,170 & 170 & & \\
\hline flute & $41,44,46,77,84,87,121,132,183,184$ & 183 & & $121,183,184$ \\
\hline piccolo & 132 & & & \\
\hline reed & 170 & 170 & & \\
\hline oboe & $41,44,46,84$ & & & \\
\hline clarinet & $41,44,46,84,132$ & & & \\
\hline bassoon & $38,39,41,44,46,66,84$ & 38 & & 39 \\
\hline saxophone & $41,44,84,132$ & & & \\
\hline brass & $\begin{array}{l}30,41,44-46,54,60,65,66,71,76,85,86 \\
119,122,125,129-131,135,138,158\end{array}$ & $71,85,122,130$ & 146 & $68,85,114$ \\
\hline high brass/small mouthpiece & 85,170 & 170 & & \\
\hline trumpet & $41,44,46,71,73,84,132$ & & & \\
\hline French horn & $41,44,46,71,84$ & & & \\
\hline tenor & 132 & & & \\
\hline baritone & $41,44,132$ & & & \\
\hline mellophone & 132 & & & \\
\hline low brass/large mouthpiece & 85,170 & 170 & & \\
\hline trombone & $41,44,46,65,71,73,84,87,132,155$ & 155 & & \\
\hline euphonium & $41,44,84$ & & & \\
\hline tuba/sousaphone & 132 & & & \\
\hline tuba & $41,44,46,71,84$ & & & \\
\hline Non-wind & 170 & & & \\
\hline keyboards & $47,125,129,135,154$ & & & 166 \\
\hline piano/keyboard & 131 & & & \\
\hline piano/organ & 46 & & & 114 \\
\hline piano & $\begin{array}{l}41,44,69,70,73,82,102,105,127,136-138 \\
177,185\end{array}$ & $\begin{array}{l}69,82,96,136 \\
137,164\end{array}$ & 144 & $\begin{array}{l}101,102,105, \\
136,137,149, \\
150,177\end{array}$ \\
\hline organ & 70 & & & \\
\hline accordion & 73,171 & & & \\
\hline strings & $\begin{array}{l}47,76,119,122,127,129,131,135,138,154 \\
172,173,176\end{array}$ & $\begin{array}{l}79,110,122,171 \\
173\end{array}$ & 180 & 114 \\
\hline
\end{tabular}


Table 3. Instruments and instrument groups investigated - cont.

\begin{tabular}{|c|c|c|c|c|}
\hline \multirow[b]{2}{*}{ Instrument type } & \multicolumn{4}{|c|}{ Reference } \\
\hline & extent/severity & $\begin{array}{l}\text { associated factors/ } \\
\text { mechanisms }\end{array}$ & $\begin{array}{l}\text { interven- } \\
\text { tion }\end{array}$ & other \\
\hline \multicolumn{5}{|l|}{$\begin{array}{l}\text { Non-wind - cont. } \\
\text { strings - cont. }\end{array}$} \\
\hline bowed strings & $54,93,125,157$ & 157 & & \\
\hline upper strings & $30,41,44-46,60,65,66,79,83,86,124,172$ & 162,163 & & $\begin{array}{l}68,124,166 \\
168\end{array}$ \\
\hline violin & $41,44-46,87,92,108,120$ & $\begin{array}{l}79,92,108,111 \\
140,178\end{array}$ & & 92,149 \\
\hline viola & $41,44-46,87,185$ & & & \\
\hline lower strings & $30,41,44-46,60,65,66,79,83,86,172$ & & & 68,166 \\
\hline cello & $41,44,46,65,87,91,185$ & 91 & & $61-63,149,151$ \\
\hline double bass & $41,44,46,106$ & 106 & & \\
\hline ajaeng & 49,50 & 49,50 & & \\
\hline haegeum & 49,50 & 49,50 & & \\
\hline plucked strings & $54,125,129$ & & & \\
\hline harp & 46,66 & & & 114 \\
\hline tres & 73 & & & \\
\hline laúd & 73 & & & \\
\hline guitar/gambus & 131 & & & \\
\hline guitar & $41,44,46,73,156$ & 156 & & 114 \\
\hline bass guitar & 106,115 & 115 & & \\
\hline gayageum & 49,50 & 49,50 & & \\
\hline geomungo & 49,50 & 49,50 & & \\
\hline bassists $^{\mathrm{a}}$ & 73,106 & 106 & & \\
\hline percussion & $\begin{array}{l}30,41,44-46,65,66,73,86,107,119,122,125 \\
127,129-131,135,138,158,160\end{array}$ & $\begin{array}{l}107,122,130 \\
160\end{array}$ & & 114 \\
\hline membranophone percussion & 160 & & & \\
\hline snare drum & 132 & & & \\
\hline bass drum & 132 & & & \\
\hline tabla & 88 & 88 & & \\
\hline keyboard percussion & 160 & & & \\
\hline auxiliary/other percussion & 160 & & & \\
\hline cymbals & 132 & & & \\
\hline santur & 154 & & & \\
\hline \multicolumn{5}{|l|}{ Mixed groupings } \\
\hline percussion/harp/keyboard & 60 & & & 68 \\
\hline harp/piano/organ/guitar & 46 & & & \\
\hline
\end{tabular}


Table 3. Instruments and instrument groups investigated - cont.

\begin{tabular}{|c|c|c|c|c|c|}
\hline \multirow[b]{2}{*}{ Instrument type } & \multicolumn{5}{|c|}{ Reference } \\
\hline & & extent/severity & $\begin{array}{l}\text { associated factors/ } \\
\text { mechanisms }\end{array}$ & $\begin{array}{l}\text { interven- } \\
\text { tion }\end{array}$ & other \\
\hline \multicolumn{6}{|l|}{ Mixed groupings - cont. } \\
\hline piano/guitar/percussion & 93 & & & & \\
\hline percussion/keyboard & 54 & & & & \\
\hline $\begin{array}{l}\text { clarinet/oboe/bassoon/ } \\
\text { trumpet/recorder/keyboard/ } \\
\text { piano/percussion }\end{array}$ & 93 & & & & \\
\hline $\begin{array}{l}\text { violin/cello/double bass/ } \\
\text { trombone/flute/guitar }\end{array}$ & 93 & & & & \\
\hline $\begin{array}{l}\text { bassoon/clarinet/French horn/ } \\
\text { oboe/tuba }\end{array}$ & 83 & & & & \\
\hline flute/trumpet/trombone & 83 & & & & \\
\hline $\begin{array}{l}\text { viola/violin/daegeum/flute/ } \\
\text { trombone/trumpet/harp/ } \\
\text { percussion }\end{array}$ & 182 & & & & \\
\hline $\begin{array}{l}\text { cello/double bass/clarinet/ } \\
\text { saxophone/bassoon/oboe/ } \\
\text { horn/tuba/piano }\end{array}$ & 182 & & & & \\
\hline $\begin{array}{l}\text { violin/viola/vocal/trombone/ } \\
\text { tuba/clarinet/saxophone }\end{array}$ & 74 & & & & \\
\hline
\end{tabular}

Mehrparvar et al. [154] also included 3 other instruments in a graph regarding prevalence, but these were not labeled. Some studies had "others" groups where the instruments were not specified. These have not been included here.

a The item "Bassists" refers to double bassists and bass guitarists, and therefore crosses the bowed and plucked string categories.

cal or eurhythmics teachers [93] were also investigated. In most cases, the musicians themselves were asked about the presence of symptoms; however, Ackermann and Driscoll [175] surveyed parents of child instrumentalists only, while McKechnie and Jacobs [177] surveyed child pianists, their parents and piano teachers.

Eight unique studies compared the prevalence of MSS and/or the consequences experienced by musicians with other groups, comparing especially the following: children playing the accordion with non-musical children [171], adult musicians with the general population [161], university music students with university medical [53-55] or health students [123], choristers with the general population [75], professional orchestral musicians with the general working population [28], professional pianists and organists with those without increased foot activity [70], and university orchestral students with those who had not played any music for at least the past year [116]. Kok et al. [54,55] also compared the medical students who did and did not play a musical instrument. Overall, the comparison between musicians and non-musicians remains under-investigated.

Most primary extent studies investigated prevalence of MSS outcomes, with only 5 studies reporting the incidence $[68,89,90,117,174]$. These, however, appeared to investigate episodic incidence, rather than true incidence. Four of these studies $[89,90,117,174]$ investigated marching or military band musicians, while the remaining one [68] investigated professional orchestral musicians. 
Data were collected by asking for daily injury reports [117], or using data from onsite or military clinics $[68,89,90,174]$. It should be noted that there were a number of other studies indicating that they were reporting incidence, whereas their methods and results indicated that they were actually reporting prevalence $[66,69,80,84,121,170]$.

The particular outcomes investigated in the studies focusing on the extent of MSS were predominantly symptoms in general $(87 \%)$ [28-30,38,40-50,53-55,60,64-66,69-88, 91-95,102,105-109,115-126,129-131,133-135,138,139, 154-161,170-173,176,177,181-183,185]. Of the studies investigating the extent of MSS, $19 \%$ reported the perceived risk or aggravating factors for symptoms, with the most common factor type relating to musical activity (Table 4), and $58 \%$ investigated consequences, with musical consequences being the most commonly reported category (Table 5).

\section{Associated factors}

Similar to the extent/severity studies, most of the studies (69\%) of associated factors/mechanisms focused on professional and/or university musicians, with orchestral and classical musicians (30\%) being the focus where the genre or ensemble was reported (Table 2). A smaller range of instruments were investigated when compared with the extent/severity studies (Table 3), with piano (8\%) and violin $(8 \%)$ being the most commonly investigated groups. One study investigated vocalists [139]. Additionally, Zamorano et al. [97] conducted a study exploring the relationship between pain and tactile thresholds, and pressure and thermal pain sensitivity in 3 groups, i.e., musicians with no pain, musicians with chronic low back pain, and nonmusicians with chronic low back pain.

Of the 74 unique primary studies investigating MSS associated factors or mechanisms, most were crosssectional studies. Exceptions were a prospective cohort study comparing MSS before and after an orchestra camp [153], and another that investigated the reported injuries and illnesses during a band camp [174]. There were also 12 case-control studies [96,97,110,111,115,140, 141,162-165,178]. Of these case-control studies, 3 included a playing-task [111,115,178], 1 resisted finger extension/flexion [165], and another emotionally and physically stressful tasks [110]. Kuppens et al. [110] used a randomized cross-over design regarding the order of emotionally or physically stressful tasks, while Woldendorp et al. [115] compared pain intensity before and after a playing task, as well as muscle activity before, during and after a playing task.

Studies of risk factors for MSS outcomes most commonly investigated activities and behaviors, particularly musical factors (72\%), while personal factors (65\%), such as age and gender, were also commonly investigated. Only 1 study investigated the relationship between the equipment used, in this case footwear, and MSS outcomes (Table 6). Some 22 studies also investigated the association between different MSS outcomes.

Similar to the extent/severity studies, the majority $(86 \%)$ of the studies of risk factors reported general symptom outcomes [27,28,30,38,40-50,53,54,60,64,65,69,71,74,76, 79-88,91-93,95-97,106-108,110,111,115,116,119,122,125, 126,129-131,133-135,138-142,154-156,160-164,170,172, $173,178,182,183]$. Two studies of instrumental musicians used perceived risk/aggravating factors as the outcomes, these being playing [64] and band [90]. Musculoskeletal symptoms consequences were the investigated outcome in $41 \%$ of the risk factor studies; however, the range of consequences was less diverse than in the extent/severity studies (Table 5).

\section{Intervention studies}

Professional and university musicians were again the most commonly investigated populations $(88 \%)$, with only 2 studies investigating children [179,180] (Table 2). Orchestral musicians were again the most frequently investigated type of musicians (44\%) (Table 2). Unlike the 
Table 4. Perceived risk or aggravating factors

\begin{tabular}{|c|c|}
\hline Factor & Reference \\
\hline \multicolumn{2}{|l|}{ Music-related } \\
\hline Playing & $64,68,73,102,124,175$ \\
\hline military band & 90 \\
\hline marching band & 132 \\
\hline Work & 66 \\
\hline long sessions of playing & $29,66,121,127$ \\
\hline extensive practicing & 129 \\
\hline change in practice routine & 137 \\
\hline sudden increase or decrease in playing hours & 29 \\
\hline sudden increase in playing time & 121 \\
\hline increase in playing time & 30 \\
\hline sudden increase in playing ${ }^{\mathrm{a}}$ & 66,127 \\
\hline insufficient warm-up & $29,66,121$ \\
\hline insufficient rest breaks & 121,129 \\
\hline too few breaks when playing & 29,94 \\
\hline technique flaws & $29,66,121$ \\
\hline inappropriate technique and/or abnormal body posture & 129 \\
\hline increased difficulty & 30 \\
\hline particular repertoire or a difficult piece & 29 \\
\hline repertoire scheduling & 66 \\
\hline playing when physically exhausted & 29 \\
\hline carrying an instrument or other equipment & 29 \\
\hline variation in the functioning of and/or malfunction of the instrument & 29 \\
\hline instrument set up & $66,121,127$ \\
\hline conductor approach & 66 \\
\hline touring/travelling & 66,121 \\
\hline continuing to play with symptoms & 94 \\
\hline performing the same movement repetitively & 94 \\
\hline \multicolumn{2}{|l|}{ Dance-related } \\
\hline dance partner & 139 \\
\hline difficult choreography or movement pattern & 139 \\
\hline too many repetitions/rehearsals & 139 \\
\hline \multicolumn{2}{|l|}{ Environmental } \\
\hline inadequate chairs & $29,30,121$ \\
\hline cramped playing conditions & 29 \\
\hline practice/rehearsal venue & 85 \\
\hline climatic conditions & 139 \\
\hline
\end{tabular}


Table 4. Perceived risk or aggravating factors - cont.

\begin{tabular}{|c|c|}
\hline Factor & Reference \\
\hline \multicolumn{2}{|l|}{ Environmental - cont. } \\
\hline temperature & 29 \\
\hline lighting & 29 \\
\hline dance floor & 139 \\
\hline changing dance floors & 139 \\
\hline dance floor too slippery or too sticky & 139 \\
\hline \multicolumn{2}{|l|}{ Psychosocial } \\
\hline emotional problems & 30 \\
\hline stress and/or anxiety & 29 \\
\hline mental stress & 139 \\
\hline stress & 66,127 \\
\hline performance anxiety & 66,121 \\
\hline depression & 29 \\
\hline feelings of inadequacy & 29 \\
\hline job dissatisfaction & 29 \\
\hline time pressure/practicing with a deadline & 29 \\
\hline own performance aspiration & 139 \\
\hline pressure by the school & 139 \\
\hline pressure by the teachers & 139 \\
\hline parents' expectations & 139 \\
\hline lack of support from the manager/conductor & 29 \\
\hline lack of social support & 29 \\
\hline other psychological causes & 139 \\
\hline \multicolumn{2}{|l|}{ Physical condition } \\
\hline physiological strains & 121 \\
\hline lack of endurance or strength & 29 \\
\hline poor physical condition & 121 \\
\hline poor flexibility & 66,121 \\
\hline poor fitness & 66,139 \\
\hline excess muscle tension & $29,66,127$ \\
\hline poor posture & $29,30,66,121,127$ \\
\hline fatigue & 139 \\
\hline physical fatigue & 139 \\
\hline muscle fatigue & 66 \\
\hline general overload situation & 139 \\
\hline
\end{tabular}


Table 4. Perceived risk or aggravating factors - cont.

\begin{tabular}{ll}
\hline \multicolumn{1}{c}{ Factor } & Reference \\
\hline Other & \\
lack of understanding of physical strain & 121 \\
lack of basic knowledge of anatomy/physiology & 129 \\
loss of concentration & 139 \\
insufficient rest & 66,127 \\
poor injury management & 66 \\
inadequate nutrition & 139 \\
bad drinking habits & 139 \\
another student & 139 \\
ignoring warning signals & 139 \\
\hline
\end{tabular}

Chimenti et al. [29] also grouped their factors as playing-related, musculoskeletal, work environment-related and psychosocial.

${ }^{a}$ Unclear which parameter (e.g., time, intensity) is increased.

Table 5. Consequences of the musculoskeletal symptoms investigated, by study type

\begin{tabular}{llll}
\hline \multirow{2}{*}{ Musculoskeletal symptoms consequences } & \multicolumn{1}{c}{ Reference } \\
\cline { 2 - 3 } & & $\begin{array}{c}\text { associated factors/ } \\
\text { mechanisms }\end{array}$ & intervention \\
\hline Seeking advice/treatment/informing others & & \\
professional care & $29,39,134$ & \\
hospitalization & 119 & \\
military or onsite clinic & $68,89,90$ & \\
doctor/physiotherapist/chiropractor & 119 & \\
or similar professional & & $28,38,43,174$ \\
medical/health professionals & $28,38,43,53,102,126$, & \\
& 174,183 & 38 \\
medical practitioner & $30,38,39,69,94,124$, & \\
& $129,139,183,184$ & \\
specialist & $28,53,74,139$ & \\
surgeons & 102 & \\
orthopedist & 92,124 & \\
physician & 85 & \\
general practitioners & $28,53,102,124$ & \\
physiotherapists & $28,30,39,53,69,94,102$, & \\
& $121,128,129,183$ & \\
physiotherapist/occupational therapist & 92 & \\
occupational therapy & 30 & \\
occupational therapists/acupuncturists & 102 & \\
acupuncturist & 39 & \\
\hline
\end{tabular}


Table 5. Consequences of the musculoskeletal symptoms investigated, by study type - cont.

\begin{tabular}{|c|c|c|c|}
\hline \multirow[b]{2}{*}{ Musculoskeletal symptoms consequences } & \multicolumn{3}{|c|}{ Reference } \\
\hline & extent/severity & $\begin{array}{l}\text { associated factors/ } \\
\text { mechanisms }\end{array}$ & intervention \\
\hline \multicolumn{4}{|l|}{ Seeking advice/treatment/informing others - cont. } \\
\hline massage therapists & $39,121,183$ & & \\
\hline chiropractors & $\begin{array}{l}28,30,39,69,102,121 \\
128,183\end{array}$ & & \\
\hline osteopath & $39,121,128$ & & \\
\hline psychologists or counselors & 102 & & \\
\hline alternative medicine & $30,53,94$ & & \\
\hline Alexander practitioner & $30,39,94,121,128$ & & \\
\hline Feldenkrais practitioner & $30,39,128$ & & \\
\hline Bowen therapy & 128 & & \\
\hline yoga teacher & $39,121,128$ & & \\
\hline Pilates instructor & 39,128 & & \\
\hline school administration & 139 & & \\
\hline teachers & 69,139 & & \\
\hline instrumental tutors & 39,129 & & \\
\hline other tutors & 129 & & \\
\hline superiors & 85 & & \\
\hline colleagues & 85,129 & & \\
\hline friends & 129,139 & & \\
\hline the Internet & 129 & & \\
\hline attempted to conceal the problem & 85 & & \\
\hline \multicolumn{4}{|l|}{ Treatments } \\
\hline self-treatment & 38 & 38 & \\
\hline general body training & 128 & & \\
\hline exercises/stretching & 129 & & \\
\hline stretching & $39,121,128$ & & \\
\hline exercise or posture program & 94 & & \\
\hline exercise & 39,121 & & \\
\hline muscle resistance exercises & 128 & & \\
\hline braces/splints/orthoses & 94 & & \\
\hline brace & 39 & & \\
\hline hand splint & 129 & & \\
\hline bandages & 129 & & \\
\hline heat & $39,121,128$ & & \\
\hline
\end{tabular}


Table 5. Consequences of the musculoskeletal symptoms investigated, by study type - cont.

\begin{tabular}{|c|c|c|c|}
\hline \multirow[b]{2}{*}{ Musculoskeletal symptoms consequences } & \multicolumn{3}{|c|}{ Reference } \\
\hline & extent/severity & $\begin{array}{l}\text { associated factors/ } \\
\text { mechanisms }\end{array}$ & intervention \\
\hline \multicolumn{4}{|l|}{ Treatments - cont. } \\
\hline ice & $39,121,128$ & & \\
\hline taking medication & $\begin{array}{l}43,94,119,128,129,139 \\
183\end{array}$ & 43 & \\
\hline anti-inflammatory medication & $39,115,121,183$ & & \\
\hline cortisone injection & 128 & & \\
\hline pain killers & $28,39,115,121,131,183$ & 28 & \\
\hline $\mathrm{gel} / \mathrm{cream}$ & 129 & & \\
\hline rest & $39,121,129$ & & \\
\hline relaxation techniques & 128 & & \\
\hline warm up & 128 & & \\
\hline massage & 128 & & \\
\hline surgery & 94 & & \\
\hline diagnostic imaging & 94 & & \\
\hline \multicolumn{4}{|l|}{ Impact on work or study } \\
\hline time off from work/studies & $\begin{array}{l}28,64,66,85,95,119 \\
139\end{array}$ & 28 & \\
\hline missed opportunities for work/study & 128 & & \\
\hline change in jobs/duties & 119 & & \\
\hline prevented from doing normal work & 119 & & \\
\hline hindered ability to perform their work & 106 & & \\
\hline workers' compensation claim & 29 & 29 & \\
\hline \multicolumn{4}{|l|}{ Impact on the quality of musical activity } \\
\hline impaired playing ability/interferes with playing & $\begin{array}{l}27,28,30,38-44,64,66 \\
73,76,82,86,119,125 \\
127,128,130,136,137 \\
153,172,176\end{array}$ & $\begin{array}{l}27,28,38,40-44,64 \\
82,86,127,130,136 \\
137,153,165,172\end{array}$ & 99,100 \\
\hline difficulty using the usual technique & 119,176 & & \\
\hline difficulty playing as well as they wanted to & 119,176 & & \\
\hline difficulty playing the usual amount of time & 119,176 & & \\
\hline distracted while playing & 131,183 & & \\
\hline affects the high range & 71,85 & 71,85 & \\
\hline affects the low range & 71,85 & 71 & \\
\hline playing flexibility & 71 & 71 & \\
\hline affects transition from the low to the high range & 71,85 & 71 & \\
\hline affects transition from the high to the low range & 71,85 & 71 & \\
\hline
\end{tabular}


Table 5. Consequences of the musculoskeletal symptoms investigated, by study type - cont.

\begin{tabular}{|c|c|c|c|}
\hline \multirow[b]{2}{*}{ Musculoskeletal symptoms consequences } & \multicolumn{3}{|c|}{ Reference } \\
\hline & extent/severity & $\begin{array}{l}\text { associated factors/ } \\
\text { mechanisms }\end{array}$ & intervention \\
\hline \multicolumn{4}{|l|}{ Impact on the quality of musical activity - cont. } \\
\hline affects moving in both directions & 71 & 71 & \\
\hline affects the ability to hold long notes ("shaking") & 71,85 & 71 & \\
\hline affects tone quality & 71,85 & 71 & \\
\hline $\begin{array}{l}\text { affects the beginning of notes (attack } \\
\text { "blockage") }\end{array}$ & 71,85 & 71 & \\
\hline affects accuracy ("cracked" notes) & 71,85 & 71 & \\
\hline breaking off held notes & 71,85 & 71 & \\
\hline affects attack in tongued passages & 71,85 & 71 & \\
\hline affects coordination & 71,85 & 71 & \\
\hline affects the marching technique & 130 & 130 & \\
\hline \multicolumn{4}{|l|}{ Changes made to musical activity } \\
\hline technique change & 94 & & \\
\hline regular practice breaks & 128 & & \\
\hline $\begin{array}{l}\text { stopped playing with discomfort/increase } \\
\text { in discomfort }\end{array}$ & 128,129 & & \\
\hline changed postural playing position & 69,105 & & \\
\hline increased playing time & 39 & & \\
\hline warm up & 128 & & \\
\hline reduced playing time & $\begin{array}{l}39,94,121,128,131,183 \\
184\end{array}$ & & \\
\hline affected playing time & 30 & & \\
\hline stopped playing/time off from playing ${ }^{\mathrm{a}}$ & $\begin{array}{l}69,82,94,102,128,130 \\
137,154,183\end{array}$ & 82,130 & \\
\hline cannot play & 30,155 & 155 & 112 \\
\hline paused from practicing & 28 & 28 & \\
\hline paused from rehearsals & 28 & 28 & \\
\hline omitted playing at concerts & 28 & 28 & \\
\hline missed playing commitments & 128 & & \\
\hline missed rehearsals/services & 29,159 & & \\
\hline missed competitions & 159 & & \\
\hline stopped marching participation & 130 & 130 & \\
\hline absence from orchestra & 30 & & \\
\hline cannot give classes & 30 & & \\
\hline missed a scheduled practice/performance & 132 & & \\
\hline music disability scales & $133,153,157$ & $\begin{array}{l}133,141,153,157 \\
159,162\end{array}$ & \\
\hline
\end{tabular}


Table 5. Consequences of the musculoskeletal symptoms investigated, by study type - cont.

\begin{tabular}{|c|c|c|c|}
\hline \multirow[b]{2}{*}{ Musculoskeletal symptoms consequences } & \multicolumn{3}{|c|}{ Reference } \\
\hline & extent/severity & $\begin{array}{l}\text { associated factors/ } \\
\text { mechanisms }\end{array}$ & intervention \\
\hline \multicolumn{4}{|l|}{ Impact on life outside of work, study and music } \\
\hline $\begin{array}{l}\text { functional limitations/impact on life/daily } \\
\text { activities }\end{array}$ & $53,55,90,127,130$ & $80,90,130$ & \\
\hline $\begin{array}{l}\text { impaired level of function with activities } \\
\text { of daily living at home }\end{array}$ & 28 & 28 & \\
\hline impact on function of leisure time activities & 28 & 28 & \\
\hline impact on sleep & 28,124 & 28 & \\
\hline emotional impact & 55 & & \\
\hline financial problems & 128 & & \\
\hline social isolation & 128 & & \\
\hline opening a tight or new jar & 138 & & \\
\hline $\begin{array}{l}\text { recreational activities in which external force } \\
\text { impact the upper extremity }\end{array}$ & 138 & & \\
\hline general disability scale ${ }^{b}$ & $86,119,133,138,157$ & $\begin{array}{l}80,86,97,119,133 \\
138,141,157,162\end{array}$ & 113 \\
\hline
\end{tabular}

One study referred to time off, but did not specify whether this concerned the time off from playing, work or study [131].

In some cases (e.g., [127,128] certain consequences were combined, e.g., the impact on playing and financial problems, and Artigues-Cano et al. [184]), the prevalence of those who had injuries related to flute playing or who sought medical advice for their pain was reported.

${ }^{a}$ Based on the report of how many musicians continued to play with symptoms or a worsening of symptoms in 3 studies [39,102,137].

${ }^{\mathrm{b}}$ The scale used by Berque et al. [86] incorporated musical and general disability items.

Table 6. Summary of the factors associated with musculoskeletal symptom outcomes

\begin{tabular}{|c|c|}
\hline & Reference \\
\hline \multicolumn{2}{|c|}{ Personal (non-modifiable) } \\
\hline gender/sex & $\begin{array}{l}27,28,30,38,40-47,49,50,53,69,71,74,76,79,80,82,84, \\
86,90,91,93,95,97,106-108,116,119,127,129,131, \\
134-139,141,142,154,155,157,162,170,172,173,182,183\end{array}$ \\
\hline age & $\begin{array}{l}27,30,40-46,49,50,69,71,74,76,79,82,84,86,90,95,97, \\
106-108,119,129,134,136,137,141,142,154,157,162,172, \\
182\end{array}$ \\
\hline hand dominance & $82,97,154$ \\
\hline race & 82,90 \\
\hline \multicolumn{2}{|l|}{ Physical } \\
\hline \multicolumn{2}{|l|}{ non musical } \\
\hline general health & 106 \\
\hline physical health & 133 \\
\hline medical history & 80 \\
\hline
\end{tabular}


Table 6. Summary of the factors associated with musculoskeletal symptom outcomes - cont.

\begin{tabular}{|c|c|}
\hline Factor & Reference \\
\hline \multirow{2}{*}{\multicolumn{2}{|c|}{$\begin{array}{l}\text { Physical - cont. } \\
\text { non musical - cont. }\end{array}$}} \\
\hline & \\
\hline other health conditions & 82,174 \\
\hline body mass index & $49,50,80,82,90,119,133,141,154,162,170,172$ \\
\hline height & $49,50,82$ \\
\hline weight & $49,50,82$ \\
\hline other anthropometric factors & $69,82,96,136,155,164,172$ \\
\hline trigger point/pressure pain threshold & $27,60,65,91,96,97,110,163$ \\
\hline tactile thresholds & 97 \\
\hline hypermobility & $60,82,172$ \\
\hline range of motion & $60,82,178$ \\
\hline rotation speed & 82 \\
\hline muscle resistance/strength tests & 60,82 \\
\hline craniocervical flexion test & 162 \\
\hline nerve root/peripheral nerve tests & 60 \\
\hline motor performance tests & 163 \\
\hline kinesthetic awareness & 141 \\
\hline fine motor dexterity & 141 \\
\hline sensorimotor incongruence coordination & 140 \\
\hline myoelectric activity & 178 \\
\hline palpation soreness/pain & 60 \\
\hline push-ups & 90 \\
\hline sit-ups & 90 \\
\hline 2 mile run & 90 \\
\hline cortisol levels & 81 \\
\hline intracellular $\mathrm{pH}$ & 165 \\
\hline phosphocreatine levels & 165 \\
\hline inorganic phosphate levels & 165 \\
\hline $\mathrm{Pi} / \mathrm{PCr}$ ratio & 165 \\
\hline fatigue & 82 \\
\hline \multicolumn{2}{|l|}{ musical } \\
\hline chin rest sore & 82 \\
\hline weight of the instrument & 80 \\
\hline self-reported muscle tension when playing & 137 \\
\hline seat height when playing & 137 \\
\hline self-reported playing posture & $69,116,137,154$ \\
\hline
\end{tabular}


Table 6. Summary of the factors associated with musculoskeletal symptom outcomes - cont.

\begin{tabular}{|c|c|}
\hline Factor & Reference \\
\hline \multicolumn{2}{|l|}{ Physical - cont. } \\
\hline \multicolumn{2}{|l|}{ musical - cont. } \\
\hline maximum interval on the piano & 82 \\
\hline incorrect embouchure & 116 \\
\hline strong resistance & 116 \\
\hline pressure from mouthpiece & 116 \\
\hline loss of lip & 82 \\
\hline loss of seal & 82 \\
\hline standing time & 90 \\
\hline marching time & 90 \\
\hline range of neck motion while playing & 178 \\
\hline playing-related perceived exertion & 111 \\
\hline muscle activity before, while and/or after playing & 111,115 \\
\hline \multicolumn{2}{|l|}{ Psychosocial/organizational } \\
\hline \multicolumn{2}{|l|}{ non-musical } \\
\hline marital status & 82,90 \\
\hline number of children & 82 \\
\hline part-time employment & 139 \\
\hline employment duration & 154 \\
\hline perceived job security & 95 \\
\hline perceived physical environment & 80 \\
\hline work psychosocial factors & 80 \\
\hline support at work & 95 \\
\hline control at work & 95 \\
\hline career satisfaction & 107,134 \\
\hline goals achievement & 107 \\
\hline mental health & $119,133,162$ \\
\hline stress & $79,160,173$ \\
\hline psychological disturbance/distress & 92,106 \\
\hline somatizing tendency & 95 \\
\hline mood & 95 \\
\hline depression & $27,65,82,97,142$ \\
\hline anxiety & $27,82,97,142$ \\
\hline pain-related anxiety & 134 \\
\hline personality type & 159 \\
\hline self-esteem & 27 \\
\hline
\end{tabular}


Table 6. Summary of the factors associated with musculoskeletal symptom outcomes - cont.

\begin{tabular}{|c|c|}
\hline Factor & Reference \\
\hline \multicolumn{2}{|l|}{ Psychosocial/organizational - cont. } \\
\hline \multicolumn{2}{|l|}{ non-musical - cont. } \\
\hline generalized self-efficacy & 27 \\
\hline neuroticism & 27 \\
\hline locus of control & 27 \\
\hline positive affect & 134 \\
\hline negative affect & 134 \\
\hline financial situation & 139 \\
\hline \multicolumn{2}{|l|}{ musical } \\
\hline enjoyment of music/playing & 40,173 \\
\hline $\begin{array}{l}\text { information from the teacher on reducing playing-related } \\
\text { musculoskeletal disorder risk }\end{array}$ & 137 \\
\hline age when deciding to become a professional musician & 71 \\
\hline acceptability of the "no pain, no gain" attitude & 136 \\
\hline medicine taken for performance anxiety & 40 \\
\hline performance anxiety/stage fright & $27,40,45,46,65,82,95,108,134,135,172$ \\
\hline playing-related stress & 137 \\
\hline \multicolumn{2}{|l|}{ Activities/behaviors } \\
\hline \multicolumn{2}{|l|}{ non-musical } \\
\hline current healthy behaviors & 125 \\
\hline smoking & $49,50,80,95,106$ \\
\hline diet & 107 \\
\hline drinking & 49,50 \\
\hline household chores & 80 \\
\hline hobbies & $49,50,80$ \\
\hline stretching & 50 \\
\hline stretching/warming up & 49 \\
\hline prevention exercises & 107 \\
\hline physical activity/exercise & $\begin{array}{l}42,47-50,76,79,80,82,90,106,107,119,131, \\
133-136,154,173,183\end{array}$ \\
\hline relaxation techniques & 107 \\
\hline sleep & $79,82,173$ \\
\hline travel & 82 \\
\hline hand-related activities & 82 \\
\hline computer use & 42,106 \\
\hline television/video & 42 \\
\hline writing & 42 \\
\hline
\end{tabular}


Table 6. Summary of the factors associated with musculoskeletal symptom outcomes - cont.

\begin{tabular}{|c|c|}
\hline Factor & Reference \\
\hline \multicolumn{2}{|l|}{ Activities/behaviors - cont. } \\
\hline \multicolumn{2}{|l|}{ non-musical - cont. } \\
\hline electronic games & 42 \\
\hline intensive hand activities & 42 \\
\hline oral parafunction & 182 \\
\hline night bruxism & 79 \\
\hline day bruxism & 79 \\
\hline physical activities in a typical work day & 95 \\
\hline \multicolumn{2}{|l|}{ musical } \\
\hline instrument & $41,44,65,87,106,157,173$ \\
\hline instrument group & $\begin{array}{l}27,28,30,44-47,54,74,76,79,80,83,85,86,93 \\
95,129,134,135,138,142,154,170,172,182\end{array}$ \\
\hline number of instruments played & 41,44 \\
\hline whether the piano was their main instrument & 69 \\
\hline playing other instruments ( $\geq 5 \mathrm{~h} /$ week) & 106 \\
\hline chin rest type & 108 \\
\hline age of starting music & $71,82,97,107,136$ \\
\hline years of playing & $\begin{array}{l}40,44,47,48,69,74,82,85,86,92,97,108,116 \\
119,134-137,157,162,170,173,182\end{array}$ \\
\hline years in the profession & $45,49,50,71,80,84,86$ \\
\hline years of university study/ensemble experience & $71,85,134$ \\
\hline education level & $53,69,90,137,139,173$ \\
\hline which university/academy they attended & 54,127 \\
\hline professional vs. university & 91 \\
\hline teachers vs. performers vs. players & 69 \\
\hline professionals vs. non-professionals & 69 \\
\hline music major vs. non-major & 130 \\
\hline active duty vs. air national guard & 85 \\
\hline main musical activity (e.g., orchestral) & 107 \\
\hline orchestra type (pit vs. stage vs. pit/stage) & 64 \\
\hline military performance group & 90 \\
\hline playing genre & $106,127,137$ \\
\hline repertoire difficulty & 40 \\
\hline playing in additional ensembles & 173 \\
\hline changes to embouchure technique & 71 \\
\hline changes to breathing technique & 71 \\
\hline type of playing technique & 69,106 \\
\hline
\end{tabular}


Table 6. Summary of the factors associated with musculoskeletal symptom outcomes - cont.

\begin{tabular}{|c|c|}
\hline Factor & Reference \\
\hline \multirow{2}{*}{\multicolumn{2}{|c|}{$\begin{array}{l}\text { Activities/behaviors - cont. } \\
\text { musical - cont. }\end{array}$}} \\
\hline & \\
\hline general workload per week & 71 \\
\hline duration and/or frequency of musical activity & $\begin{array}{l}40,41,44,47,48,69,71,74,76,80,82,83,85,86, \\
93,96,97,106-108,116,119,127,129,134,136, \\
137,154,156,157,162,1709,172,173,182\end{array}$ \\
\hline set amount of musical activity & 85 \\
\hline changes in the amount of playing time & $40,153,172$ \\
\hline warm ups & $40,71,80,82,122,137,173$ \\
\hline cool downs & 40 \\
\hline breaks & $40,69,80,82,136,137$ \\
\hline stop practice with playing fatigue & 82 \\
\hline stop practice with mental fatigue & 82 \\
\hline public concerts & 82,107 \\
\hline resting time before a concert & 107 \\
\hline day of a band camp & 174 \\
\hline upper limb physical requirements & 80 \\
\hline \multirow{3}{*}{\multicolumn{2}{|c|}{$\begin{array}{l}\text { Equipment } \\
\text { musical } \\
\text { shoe }\end{array}$}} \\
\hline & \\
\hline & \\
\hline heel fit & 90 \\
\hline width & 90 \\
\hline toe room & 90 \\
\hline cushioning & 90 \\
\hline breathability & 90 \\
\hline durability & 90 \\
\hline \multicolumn{2}{|l|}{ feet } \\
\hline too hot in warm weather & 90 \\
\hline to cold in cold weather & 90 \\
\hline orthotics & 90 \\
\hline frequency of replacing shoes & 90 \\
\hline Association between musculoskeletal symptom outcomes & $\begin{array}{l}29,42,44,46,71,74,76,82,86,88,96,106,126 \\
129,133,134,138,141,155,157,162,172,174\end{array}$ \\
\hline
\end{tabular}

other study types, few were specific to an instrument or instrument group, with 1 investigating brass instrumentalists [146], and another one pianists [144] (Table 3).
Only 16 unique intervention studies were identified. The study designs included randomized controlled trials [58,59,113,143], a randomized cross-over study [144], 
non-randomized prospective concurrent comparative studies [98,99,148,179,180], and case series/pre-post studies [68,90,100,112,145-147,149]. While Kava et al.'s [148] and Lee et al.'s [145] were intended as non-randomized prospective concurrent comparative studies, and a randomized controlled trial, respectively, the analyses reflect pre-post designs.

Three clinical studies included only symptomatic participants using radial shockwave therapy of trigger points [113], oral splints [112], or Tunia exercises [58,59]. The remaining 13 studies focused on public health interventions that included exercise programs (yoga lifestyle [98], yoga [98,179], stretches during rehearsals [180], breathing exercises [145], trunk and upper extremity endurance exercises [148], Pilates [148], and general muscle exercises [99,100,145]), education programs [143,146,147], and equipment changes (ergonomically modified piano keyboard [144], and improved footwear [90]). Chan et al. [68] implemented a physio-led onsite triage clinic to examine the feasibility of this intervention, rather than effectiveness.

The most common outcome type for intervention studies was symptoms in general $(63 \%)[58,59,98,112,113,143-$ $145,148,179,180]$, while $31 \%$ of these studies investigated consequences (Table 5). Five studies had outcomes that did not relate directly to symptoms, but instead reported the following: behaviors or responses to discomfort or pain [147], descriptions of the perceived benefits of the intervention [145], perceptions of the intervention effectiveness to manage a current performance-related or non-performance-related musculoskeletal disorder injury [100], reasons for participation in the study [100], awareness of performance-related risk factors for musculoskeletal problems [146], comfort in advising students about musculoskeletal problems [146], and the likelihood of future use and usefulness, and following through with referral advice in an onsite triage clinic [68]. Chan et al. [68] also reported qualitative feedback from musicians and the physiotherapists involved.

\section{Other studies}

There were 3 main types of "other" studies, i.e., qualitative studies (6 unique studies), clinical case series (8 unique studies), and quantitative studies (23 studies). The quantitative studies categorized as "other" in this review quantified results in ways which did not fit the above study types, i.e., they reported proportions for various outcomes, listed findings without reporting the proportion or number of participants, or reported responses to open-ended questions.

As with the other types of studies, most studies (80\%) in this category concerned professional musicians and/or university music students (Table 2). In addition to a range of instrument groups being investigated (Table 3), 2 studies reported on vocalists $[104,139]$. A wide range of outcomes were reported, including the lived experience of MSS, illness perceptions, and perceived risk factors for musicians in general, not necessarily their own symptoms (Table 7). With regards to these outcomes, Rickert et al.'s [61-63] qualitative study of professional orchestral musicians also involved collecting data from orchestral managers in addition to musicians, while their study of university music students involved collecting data from students, as well as professional orchestral musicians and orchestral managers [151]. All musicians in these studies were cellists; hence, the generalizability of these findings to the population of interest is questionable. Two studies compared musicians with other populations. Andersen et al. [103] compared the culture of pain among elite sportspeople with professional musicians, and Kok et al. [55] compared the illness perceptions regarding musculoskeletal complaints between university music and medical students.

In addition to the outcomes reported in Table 7, Ajidahun and Phillips [186] used a Delphi process to determine an optimal warm up program to prevent playing-related musculoskeletal disorders, involving a range of health professionals and musicians. Parents of child instrumentalists were surveyed by Ackermann and Driscoll [175], who reported on 
Table 7. Types of musculoskeletal symptom findings reported in "other" studies

\begin{tabular}{|c|c|}
\hline Musculoskeletal symptom findings & Reference \\
\hline \multicolumn{2}{|l|}{ Quantitative data from quantitative studies } \\
\hline perceived timeline for their MSS ${ }^{\mathrm{a}}$ & 55 \\
\hline level of concern regarding their MSS ${ }^{\mathrm{a}}$ & 55 \\
\hline level of understanding regarding their MSS ${ }^{\mathrm{a}}$ & 55 \\
\hline perceived personal control over MSS ${ }^{\mathrm{a}}$ & 55 \\
\hline perceived effectiveness of planned treatment ${ }^{\mathrm{a}}$ & 55 \\
\hline changes in symptoms and disability overtime & 149 \\
\hline whether they have the "no pain, no gain" attitude & 136,150 \\
\hline whether MSS were considered a normal part of musical activity & 150 \\
\hline $\begin{array}{l}\text { whether they believed the university/school should employ a medical specialist/doctor to deal } \\
\text { with these symptoms }\end{array}$ & 129 \\
\hline $\begin{array}{l}\text { whether they had followed the advice of a health/medical professional regarding playing position, } \\
\text { to avoid injury }\end{array}$ & 183 \\
\hline whether the school allowed them to implement the advice provided by doctors to treat their symptoms & 129 \\
\hline whether they worried about playing-related pain & 150 \\
\hline whether they believed they would not play through pain & 150 \\
\hline whether they believed that playing-related pain could lead to serious injury & 150 \\
\hline whether they believed they could find a solution to playing-related pain & 150 \\
\hline tutors reaction to playing-related MSS & 129 \\
\hline whether they spoke to the band leader about injury prevention & 73 \\
\hline whether they believed physiotherapy could help manage MSS & 94 \\
\hline awareness of descriptions of pianists' injuries & 150 \\
\hline awareness of the Alexander Technique as a preventative measure or management strategy for MSS & 94 \\
\hline whether they agreed that teachers should be trained in injury prevention and management & 183 \\
\hline whether they agreed that players should be trained in injury prevention and management & 183 \\
\hline $\begin{array}{l}\text { whether they felt they had received enough information/advice during their studies, regarding injury } \\
\text { prevention and management }\end{array}$ & 183 \\
\hline whether they believed that a knowledge of basic anatomy would be beneficial in managing injuries & 183 \\
\hline $\begin{array}{l}\text { whether they took the advice of health professionals, regarding changes to the playing position } \\
\text { to prevent injury }\end{array}$ & 183 \\
\hline physical health scale ratings for those with MSS & 149 \\
\hline mental health scale ratings for those with MSS & 149 \\
\hline reasons for not claiming workers' compensation & 29 \\
\hline $\begin{array}{l}\text { the number of respondents who believe that information from a health professional would change } \\
\text { a musician's behavior }\end{array}$ & 92 \\
\hline $\begin{array}{l}\text { whether teachers advise students about performance-related musculoskeletal disorders, and recommend } \\
\text { health professionals }\end{array}$ & 102 \\
\hline $\begin{array}{l}\text { whether teachers work with health professionals to work through students' performance-related } \\
\text { musculoskeletal disorders }\end{array}$ & 102 \\
\hline
\end{tabular}


Table 7. Types of musculoskeletal symptom findings reported in "other" studies - cont.

\begin{tabular}{|c|c|}
\hline Musculoskeletal symptom findings & Reference \\
\hline \multicolumn{2}{|l|}{ Quantitative data from quantitative studies - cont. } \\
\hline what strategies they could use to prevent injuries & 73 \\
\hline sources of education for MSS prevention & 150 \\
\hline perceived prevention strategies & 183 \\
\hline perceived risk or aggravating factors & $102,150,183$ \\
\hline felt that the practice venue was not appropriate at the time problems began & 85 \\
\hline felt they had to overblow in rehearsals at the time problems began & 85 \\
\hline perceived effectiveness or satisfaction with management strategies used & $\begin{array}{l}30,94,102,105,121 \\
124,128,129,183\end{array}$ \\
\hline \multicolumn{2}{|l|}{ Qualitative studies $^{\mathrm{b}}$} \\
\hline lived experience of symptoms & $32,51,57,103$ \\
\hline lived experience of working with symptoms & 52 \\
\hline experience with rehabilitation & 63 \\
\hline impact of symptoms on individuals & 57,63 \\
\hline impact of symptoms on the Irish music community & 57 \\
\hline how musicians cope with pain & 103 \\
\hline role of the concealment of symptoms & 63 \\
\hline culture of pain, compared with elite sportspeople & 103 \\
\hline relationship between symptoms and identity & 56 \\
\hline relationship between occupation and symptoms & 51 \\
\hline why music students pursue a career in music despite the risks & 152 \\
\hline social support and collegial attitudes & 63 \\
\hline perceptions of the injury risk related to psychosocial, organizational and workplace environmental patterns & 61 \\
\hline influence of behavioral norms and attitudes, and organizational culture towards injury & 62 \\
\hline injury attitudes and behaviors ${ }^{\mathrm{c}}$ & 151 \\
\hline attitudes and perceptions regarding symptoms & 57 \\
\hline \multicolumn{2}{|l|}{ Clinical case studies } \\
\hline whether the MSS were deemed by a physiotherapist to have been preventable & 68 \\
\hline injuries affecting playing & 68 \\
\hline injuries occurred during or immediately after playing where playing was considered the main contributor & 68 \\
\hline treatment success (as determined by the clinician) & 101,167 \\
\hline referral to other professionals & 169 \\
\hline duration of time to return to playing post-surgery & 114 \\
\hline duration of time to return to work post-surgery & 114 \\
\hline whether they could return to full playing post-surgery & 114 \\
\hline $\begin{array}{l}\text { percentage of those with MSS with impaired lumbopelvic stabilization systems and scapular stabilization } \\
\text { system, and upper crossed syndrome }\end{array}$ & 166 \\
\hline perceived risk or aggravating factors & 101,113 \\
\hline
\end{tabular}


Table 7. Types of musculoskeletal symptom findings reported in "other" studies - cont.

\begin{tabular}{ll}
\hline \multicolumn{1}{c}{ Musculoskeletal symptom findings } & \multicolumn{1}{c}{ Reference } \\
\hline Clinical case studies - cont. & $68,101,113,167-169$ \\
descriptions of the symptoms or diagnosis & $101,166,169$ \\
types of management strategies used & 166 \\
perceived effectiveness or satisfaction with management strategies used & \\
Qualitative data from primarily quantitative studies & $104,137,184$ \\
perceived risk or aggravating factors & 39,184 \\
descriptions of the symptoms or diagnosis & 39,158 \\
types of management strategies used & 92 \\
how they responded to symptoms & 125 \\
perceived causes of symptoms & 125 \\
descriptions of symptoms & \\
\hline
\end{tabular}

${ }^{a}$ Compared with medical students.

${ }^{\mathrm{b}}$ These reflect the aims of these studies rather than each specific outcome.

${ }^{c}$ Within the context of a broader study looking at how tertiary music education programs equip students for careers in music.

whether they considered playing-related pain to be a normal part of playing, and which health professionals, if any, would encourage their child to see if they were experiencing pain. Both Wood [102], and McKechnie and Jacobs [177] surveyed piano teachers, looking at the teachers' perceived knowledge of diagnosis and treatment [102], their willingness to gain knowledge about performance-related MSDs [102], whether they regularly taught students with pain or discomfort experienced when playing [177], whether they taught playing techniques in the same way irrespective of whether the student complained about pain [177], and how they taught students with injuries [177].

Storms et al. [85] asked participants for any comments within their study of military brass musicians with "embouchure problems" (the embouchure refers to the positioning of the oral structures when playing a woodwind or brass instrument), with comments broadly categorized as pressure to perform, quality of performance, impact on the sense of confidence, stresses of military life leading to problems, support from superiors, meaning of embouchure-related symptoms, dystonia treatment, frustrations with medical care, and success of medical care.

\section{DISCUSSION}

This systematic mapping review is the first of its kind to explore the musicians' MSS literature. Over a 10-year period (2007-2016), 158 articles reported 138 unique studies of musicians' MSS. Despite this large number of studies, the authors identified a number of gaps in the current evidence base, in terms of populations, risk factors, interventions, outcomes and the broader impact of musicians' MSS.

\section{Geographical bias}

There appeared to be a geographical bias in the numbers of primary studies published per continent, with more than a half being from Europe $(38 \%)$ or North America (29\%). Overall, the majority of primary studies $(85 \%)$ were from high-income countries, mirroring the broader literature around the extent of MSS [187].

This geographical bias is important to consider, because there may be some differences in training, culture, working exposures, work health and safety policy, and access to health and medical consultation between countries, which may lead to differences in the prevalence, characteristics 
and impact of MSS on musicians. Even when considering culturally similar regions, such as Australia, Europe and North America, there are differences that should be explored. For instance, in Australia few universities teach their students about musculoskeletal health [188] whereas in the United States this is compulsory [189]. Similarly, there are a number of performing arts, or even musicspecific health clinics throughout North America and Europe [190], allowing musicians to obtain advice and treatment from a health professional with ample experience and knowledge of the unique needs of musicians. In contrast, in Australia this kind of access is limited [188]. While there have been both systematic reviews [17-19] and primary quantitative studies $[38,39,102,155,183]$ of the extent of MSS, which have included musicians from numerous countries, there have been no international comparisons made. The geographical differences in the prevalence, impact and management of MSS in musicians should be explored in future research.

\section{Populations investigated}

The majority (71\%) of primary studies investigated professional musicians and university music students, while adult amateur and child musicians remain under-investigated. Even within the professional musician and university music student populations there are a number of evidence gaps. The majority of studies of professional musicians have focused solely on orchestral musicians despite the fact that orchestral musicians are likely to represent a minority of all professional musicians. For instance, in Australia 6033 people indicated their primary employment was as an instrumental musician in the Census [191], yet Ackermann et al. [66] reported that there were 580 musicians employed in a professional orchestra; hence, orchestral musicians are estimated to account for approximately $10 \%$ of all professional musicians. The authors identified few studies of teachers $[69,82,93,102]$, military band musicians $[84,85,89,90]$, or professional singers $[75,104]$, and no studies reporting freelance or self-employed musicians specifically. These are important groups to consider as they have different biomechanical and psychosocial exposures compared to orchestral musicians. The impact of MSS on these musicians may also differ. A self-employed musician, given his or her lack of job security and access to worker's compensation, may lack the financial means to access MSS treatment, and/or to have time off to allow recovery from MSS. Future research of musicians' MSS should consider investigating these under-investigated groups, and make comparisons between different types of musicians, so that various groups could be appropriately targeted, and interventions developed that are specific to the risk factors faced by particular groups of musicians.

\section{Extent/severity studies}

Regarding the extent/severity studies, the authors identified 5 studies that focused on the episodic incidence of MSS [68,89,90,117,174], with none investigating true incidence. Investigating the true incidence (i.e., the first ever episode) of MSS presents a number of challenges, given the episodic and recurrent nature of pain, and issues with lifetime recall [192]. Even when incidence is investigated over a 1-year period, the high lifetime prevalence of MSS, even in children $(67 \%$ reporting symptoms while playing) [41], means that the "at risk" population is diminished. Strategies to overcome this problem may include focusing on specific body regions, e.g., the first ever MSS in the neck region, or considering new or recurrent episodes, as has been reported here [68,89,90,117,174], and deemed acceptable in other reviews of pain [192]. Despite the large number of prevalence studies identified, few statistically compared the MSS prevalence of musicians with other populations [28,53-55,70,75,116,123,161,171]. Such comparisons are required to contextualize the findings of prevalence studies. The selection of comparison groups, and the potential biases of their usage, also need to be considered. As an example, 2 of the included stud- 
ies compared university students with medical or health students [53-55,123]. These "reference" students may not be typical of university students as they have an interest in health and are likely to have a greater knowledge of MSS and the best ways to prevent and manage these symptoms, as compared to the general population of university students. More comparative studies are required to further support the claims of musicians being a "high-risk" population; however, the choice of the comparison group should be carefully considered.

\section{Associated factors and mechanisms}

A total of 74 studies looked at the factors associated with MSS outcomes in musicians, being mainly focused on professional orchestral musicians and university music students $(69 \%)$. There were a wide range of factors investigated, including non-modifiable factors such as age and gender, instrument type, psychosocial factors, and modifiable (playing-related) factors such as duration of practice or number of breaks. The majority of outcomes reported were symptoms in general ( $86 \%$ ), with only $41 \%$ of studies investigating the factors associated with the consequences of MSS. A number of important risk factors for MSS have not yet been investigated specifically in musicians, or have been insufficiently examined, such as effort-reward imbalance, job satisfaction, and illness perceptions. Future studies should aim to position themselves within the wider context of MSS, investigating the modifiable risk factors that have been identified in other populations.

\section{Intervention studies}

Relatively few intervention studies were identified, of which $81 \%$ were public health interventions, with a particular focus on exercise and education programs. There remain opportunities for investigating a wider range of interventions - ideally based on the risk factors determined with this population. While the expectations of musicians regarding clinical management have been explored [193], the types of public health interventions that musicians would like to see implemented, their facilitators and barriers, as well as expectations, have not yet been investigated. Similarly, little is known about the breadth of the public health interventions that are currently in place, particularly within the organizations that train and employ musicians.

It should also be noted that this review did not investigate the quality of the identified studies. Therefore, it cannot be presumed that the areas in which there have been a large number of studies undertaken (such as the prevalence of MSS among professional orchestral musicians) have been adequately investigated. If the quality of this evidence is found to be poor, further studies will be required to guide policy and practice.

\section{CONCLUSIONS}

While there is a reasonably large body of evidence published in 2007-2016 about musicians' MSS, there are a number of gaps in the evidence, highlighting potential areas for future research. The authors have found that non-orchestral professional musicians, non-classical university students, amateur musicians, and children are under-investigated groups of musicians, regarding MSS. Few studies compared the prevalence of MSS outcomes between musicians and non-musicians, so future research in this area would help to substantiate the claims that musicians are at high risk of MSS.

There are opportunities for research with these underinvestigated groups, as well as others, to investigate a broader range of risk factors, particularly the potentially modifiable factors such as equipment, psychosocial and organizational factors. The majority of studies about risk factors have used cross-sectional designs; hence, there needs to be a shift towards longitudinal design to improve the current understanding of the risk factors for MSS outcomes in musicians. Doing so would aid in the development of interventions to prevent and manage MSS, which 
may optimize education and exercise programs, as well as identify opportunities for a broader range of public health interventions. There is currently no evidence regarding what musicians desire and would find acceptable in terms of public health interventions, as well as facilitators and barriers.

The current evidence suggests that the issue of musicians' MSS has not been adequately addressed. Filling the identified gaps could facilitate work towards reducing the burden of musicians' MSS.

\section{ACKNOWLEDGMENTS}

The authors would like to thank Maureen Bell (The University of Adelaide) for assisting with the development of the search strategy.

JS is the recipient of an Australian Government Research Training Program Scholarship, and a SafeWork SA WHS Supplementary Scholarship (funded by the South Australian Government). SafeWork SA and the South Australian Government do not endorse the content of this material, and the views expressed herein do not represent the views of SafeWork SA or the South Australian Government.

\section{REFERENCES}

1. Vos T, Allen C, Arora M, Barber RM, Bhutta ZA, Brown A, et al. Global, regional, and national incidence, prevalence, and years lived with disability for 310 diseases and injuries, 1990-2015: A systematic analysis for the Global Burden of Disease Study 2015. Lancet. 2016;388(10053):8-14, https:// doi.org/10.1016/S0140-6736(16)31678-6.

2. Widanarko B, Legg S, Stevenson M, Devereux J, Eng A, Mannetje A, et al. Prevalence of musculoskeletal symptoms in relation to gender, age, and occupational/industrial group. Int J Ind Ergon. 2011;41(5):561-72, https://doi.org/10.1016/ j.ergon.2011.06.002.

3. Osborne A, Blake C, Fullen BM, Meredith D, Phelan J, McNamara J, et al. Risk factors for musculoskeletal disorders among farm owners and farm workers: A systematic review.
Am J Ind Med. 2012;55(4):376-89, https://doi.org/10.1002/ ajim.22001.

4. Osborne A, Blake C, Fullen BM, Meredith D, Phelen J, McNamara J, et al. Prevalence of musculoskeletal disorders among farmers: A systematic review. Am J Ind Med. 2012;55(2):143-58, https://doi.org/10.1002/ajim.21033.

5. Stanhope J, Carver S, Weinstein P. Health outcomes of beekeeping: A systematic review. J Apic Res. 2017;56(2):10011, https://doi.org/10.1080/00218839.2017.1291208.

6. Stanhope J, Carver S, Weinstein P. The risky business of being an entomologist: A systematic review. Environ Res. 2015;140:619-33, https://doi.org/10.1016/j.envres.2015. 05.025 .

7. Kim-Fine S, Woolley SM, Weaver AL, Killian JM, Gebhart JB. Work-related musculoskeletal disorders among vaginal surgeons. Int Urogynecol J. 2013;24(7):1191-200, https://doi.org/10.1007/s00192-012-1958-x.

8. Darragh AR, Huddleston W, King P. Work-related musculoskeletal injuries and disorders among occupational and physical therapists. Am J Occup Ther. 2009;63(3):351-62, https://doi.org/10.5014/ajot.63.3.351.

9. Xu YW, Cheng ASK, Li-Tsang CWP. Prevalence and risk factors of work-related musculoskeletal disorders in the catering industry: A systematic review. Work. 2013;44(2):107-16.

10. Wu SJ. Occupational risk factors for musculoskeletal disorders in musicians: A systematic review. Med Probl Perform Art. 2007;22(2):43-51.

11. Ramazzini B. Diseases that generally afflict music teachers, singers and the like. In: F. Carnevale MM, G. Moriani, editor. Works (The diseases of workers). Verona: Cierre Edizioni; 2009.

12. Medical Problems of Performing Artists [Internet]. Narberth: Medical Problems of Performing Artists; 2018 [cited 2018 Apr 10]. Archives. Available from: https://www.sciandmed.com/mppa/backissues.aspx.

13. Performing Arts Medicine Association [Internet]. Englewood: Performing Arts Medicine Association; 2018 [cited 2018 Apr 10]. About. Available from: http://www.artsmed.org/about. 
14. Aránguiz R, Chana-Cuevas P, Alburquerque D, León M. Focal dystonia in musicians. Neurologia. 2011;26(1):45-52, https://doi.org/10.1016/j.nrl.2010.09.019.

15. Altenmüller E, Jabusch HC. Focal dystonia in musicians: Phenomenology, pathophysiology, triggering factors, and treatment. Med Probl Perform Art. 2010;25(1):3-9.

16. Bragge P, Bialocerkowski A, McMeeken J. A systematic review of prevalence and risk factors associated with playingrelated musculoskeletal disorders in pianists. Occup Med (Lond). 2006;56(1):28-38, https://doi.org/10.1093/occmed/ kqi177.

17. Stanhope J, Milanese S. The prevalence and incidence of musculoskeletal symptoms experienced by flautists. Occup Med (Lond). 2016;66(2):156-63, https://doi.org/10.1093/occmed/kqv162.

18. Silva AG, Lã FMB, Afreixo V. Pain prevalence in instrumental musicians: A systematic review. Med Probl Perform Art. 2015;30(1):8-19, https://doi.org/10.21091/mppa.2015.1002.

19. Kok LM, Huisstede BM, Voorn VM, Schoones JW, Nelissen RG. The occurrence of musculoskeletal complaints among professional musicians: A systematic review. Int Arch Occup Environ Health. 2016;89(3):373-96, https://doi. org/10.1007/s00420-015-1090-6.

20. Hanon S, Rongé R, Potvin M, Schuermans D, Vincken W. Occupational asthma due to colophony in a violin player. J Allergy Clin Immunol Pract. 2014;2(5):624-5, https://doi. org/10.1016/j.jaip.2014.06.018.

21. Fuhrmann A, Wijsman S, Weinstein P, Poulsen D, Franklin P. Asthma among musicians in Australia: Is there a difference between wind/brass and other players? Med Probl Perform Art. 2009;24(4):170-4.

22. Schmidt JH, Pedersen ER, Paarup HM, Christensen-Dalsgaard J, Andersen T, Poulsen T, et al. Hearing loss in relation to sound exposure of professional symphony orchestra musicians. Ear Hear. 2014;35(4):448-60, https://doi.org/10.1097/ AUD.0000000000000029.

23. Schink T, Kreutz G, Busch V, Pigeot I, Ahrens W. Incidence and relative risk of hearing disorders in professional musicians. Occup Environ Med 2014;71(7):472-6, https:// doi.org/10.1136/oemed-2014-102172.

24. Vine K, DeLeo V. Dermatologic manifestations of musicians: A case report and review of skin conditions in musicians. Cutis. 2011;87(3):117-21.

25. Jue MS, Kim YS, Ro YS. Fiddler's neck accompanied by allergic contact dermatitis to nickel in a viola player. Ann Dermatol. 2010;22(1):88-90, https://doi.org/10.5021/ad. 2010.22.1.88

26. Gambichler T, Uzun A, Boms S, Altmeyer P, Altenmüller E. Skin conditions in instrumental musicians: A self-reported survey. Contact Derm. 2008;58(4):217-22, https://doi.org/ 10.1111/j.1600-0536.2007.01310.x.

27. Kenny D, Ackermann B. Performance-related musculoskeletal pain, depression and music performance anxiety in professional orchestral musicians: A population study. Psychol Music. 2015;43(1):43-60, https://doi.org/ $10.1177 / 0305735613493953$.

28. Paarup HM, Baelum J, Holm JW, Manniche C, Wedderkopp N. Prevalence and consequences of musculoskeletal symptoms in symphony orchestra musicians vary by gender: A cross-sectional study. BMC Musculoskelet Disord. 2011;12:223, https://doi.org/10.1186/1471-2474-12-223.

29. Chimenti RL, van Dillen LR, Prather H, Hunt D, Chimenti PC, Khoo-Summers L. Underutilization of worker's compensation insurance among professional orchestral musicians. Med Probl Perform Art. 2013;28(1):54-60.

30. Abréu-Ramos AM, Micheo WF. Lifetime prevalence of upper-body musculoskeletal problems in a professional-level symphony orchestra: Age, gender, and instrument-specific results. Med Probl Perform Art. 2007;22(3):97-104.

31. Bragge P, Bialocerkowski A, McMeeken J. Understanding playing-related musculoskeletal disorders in elite pianists: a grounded theory study. Med Probl Perform Art. 2006;21(2):71-9.

32. Guptill C. The lived experience of professional musicians with playing-related injuries: A phenomenological inquiry. Med Probl Perform Art. 2011;26(2):84-95. 
33. Kaneko Y, Lianza S, Dawson WJ. Pain as an incapacitating factor in symphony orchestra musicians in São Paulo, Brazil. Med Probl Perform Art. 2005;20(4):168-74.

34. Grant MJ, Booth A. A typology of reviews: An analysis of 14 review types and associated methodologies. Health Info Libr J. 2009;26(2):91-108, https://doi.org/10.1111/j.1471-1842. 2009.00848.x.

35. Baadjou VA, Roussel NA, Verbunt JA, Smeets RJ, de Bie RA. Systematic review: risk factors for musculoskeletal disorders in musicians. Occup Med (Lond). 2016;66(8):61422, https://doi.org/10.1093/occmed/kqw052.

36. Zaza C. Playing-related musculoskeletal disorders in musicians: A systematic review of incidence and prevalence. CMAJ. 1998;158(8):1019-25.

37. Van Mechelen W, Hlobil H, Kemper HCG. Incidence, severity, aetiology and prevention of sports injuries. A review of concepts. Sports Med. 1992;14(2):82-99, https://doi.org/ 10.2165/00007256-199214020-00002.

38. Brusky P. The high prevalence of injury among female bassoonists. Med Probl Perform Art. 2010;25(3):120-5.

39. Brusky P. High prevalence of performance-related musculoskeletal disorders in bassoon players. Med Probl Perform Art. 2009;24(2):81-7.

40. Ranelli S, Smith A, Straker L. The association of music experience, pattern of practice and performance anxiety with playing-related musculoskeletal problems (PRMP) in children learning instrumental music. Int $\mathrm{J}$ Music Educ. 2015;33(4):390-412, https://doi.org/10.1177/02557614 15597151.

41. Ranelli S, Smith A, Straker L. Playing-related musculoskeletal problems in child instrumentalists: The influence of gender, age, and instrument exposure. Int J Music Educ. 2011;29(1):28-44, https://doi.org/10.1177/0255761410389662.

42. Ranelli S, Straker L, Smith A. Soreness during non-music activities is associated with playing-related musculoskeletal problems: An observational study of 731 child and adolescent instrumentalists. J Physiother. 2014;60(2):102-8, https://doi.org/10.1016/j.jphys.2014.05.005.
43. Ranelli S, Straker L, Smith A. Prevalence of playingrelated musculoskeletal symptoms and disorders in children learning instrumental music. Med Probl Perform Art. 2008;23(4):178-85.

44. Ranelli S, Straker L, Smith A. Playing-related musculoskeletal problems in children learning instrumental music: The association between problem location and gender, age, and music exposure factors. Med Probl Perform Art. 2011;26(3):123-39.

45. Steinmetz A, Scheffer I, Esmer E, Delank KS, Peroz I. Frequency, severity and predictors of playing-related musculoskeletal pain in professional orchestral musicians in Germany. Clin Rheumatol. 2015;34(5):965-73, https://doi. org/10.1007/s10067-013-2470-5.

46. Steinmetz A, Zeh A, Delank KS, Peroz I. Symptoms of craniomandibular dysfunction in professional orchestra musicians. Occup Med (Lond). 2014;64(1):17-22, https://doi.org/ 10.1093/occmed/kqt148.

47. Nawrocka A, Mynarski W, Powerska-Didkowska A, Grabara M, Garbaciak W. Musculoskeletal pain among Polish music school students. Med Probl Perform Art. 2014;29(2): 64-9, https://doi.org/10.21091/mppa.2014.2015.

48. Nawrocka A, Mynarski W, Powerska A, Grabara M, Groffik D, Borek Z. Health-oriented physical activity in prevention of musculoskeletal disorders among young Polish musicians. Int J Occup Med Environ Health. 2014;27(1):28-37, https://doi.org/10.2478/s13382-014-0224-5.

49. Kim JY, Min SN, Cho YJ, Choi JH. The prevalence of musculoskeletal disorders of traditional Korean string instrument player. J Ergon Soc Korea. 2012;31(6):749-56, https:// doi.org/10.5143/JESK.2012.31.6.749.

50. Kim JY, Kim MS, Min SN, Cho YJ, Choi J. Prevalence of playing-related musculoskeletal disorders in traditional Korean string instrument players. Med Probl Perform Art. 2012;27(4):212-8.

51. Guptill C. Injured professional musicians and the complex relationship between occupation and health. J Occup Sci. 2012; 19(3):258-70, https://doi.org/10.1080/14427591.2012.670901. 
52. Guptill C. The lived experience of working as a musician with an injury. Work. 2011;40(3):269-80, https://doi.org/10.3233/ WOR-2011-1230.

53. Kok LM, Nelissen RG, Huisstede BM. Prevalence and consequences of arm, neck, and/or shoulder complaints among music academy students: A comparative study. Med Probl Perform Art. 2015;30(3):163-8, https://doi.org/10.21091/ mppa.2015.3031.

54. Kok LM, Vlieland TP, Fiocco M, Nelissen RG. A comparative study on the prevalence of musculoskeletal complaints among musicians and non-musicians. BMC Musculoskelet Disord. 2013;14:9, https://doi.org/10.1186/1471-2474-14-9.

55. Kok LM, Vilet Vlieland TP, Fiocco M, Kaptein AA, Nelissen RG. Musicians' illness perceptions of musculoskeletal complaints. Clin Rheumatol. 2013;32(4):487-92, https://doi. org/10.1007/s10067-013-2199-1.

56. Doherty L, Wilson IM, McKeown L. Practicing safe trad: Why existing approaches to playing-related musculoskeletal disorders may not help the Irish traditional music community. Med Probl Perform Art. 2013;28(4):181-7.

57. Wilson IM, Doherty L, McKeown L. Perceptions of playingrelated musculoskeletal disorders (PRMDs) in Irish traditional musicians: A focus group study. Work. 2014;49(4):67988. https://doi.org/10.3233/WOR-131737.

58. Sousa CM, Moreira L, Coimbra D, Machado J, Greten HJ. Immediate effects of Tuina techniques on working-related musculoskeletal disorder of professional orchestral musicians. J Integr Med. 2015;13(4):257-61, https://doi.org/ 10.1016/S2095-4964(15)60181-9.

59. Sousa CM, Coimbra D, Machado J, Greten HJ. Effects of self-administered exercises based on Tuina techniques on musculoskeletal disorders of professional orchestra musicians: A randomized controlled trial. J Integr Med. 2015;13(5): 314-8, https://doi.org/10.1016/S2095-4964(15)60194-7.

60. Paarup HM, Baelum J, Manniche C, Holm JW, Wedderkopp N. Occurrence and co-existence of localized musculoskeletal symptoms and findings in work-attending orchestra musicians - An exploratory cross-sectional study.
BMC Res Notes. 2012;5:541, https://doi.org/10.1186/17560500-5-541.

61. Rickert DL, Barrett MS, Ackermann BJ. Injury and the orchestral environment: Part I. The role of work organisation and psychosocial factors in injury risk. Med Probl Perform Art. 2013;28(4):219-29.

62. Rickert DLL, Barrett MS, Ackermann BJ. Injury and the orchestral environment: Part II. Organisational culture, behavioural norms, and attitudes to injury. Med Probl Perform Art. 2014;29(2):94-101, https://doi.org/10.21091/ mppa.2014.2020.

63. Rickert DLL, Barrett MS, Ackermann BJ. Injury and the orchestral environment: Part III. The role of psychosocial factors in the experience of musicians undertaking rehabilitation. Med Probl Perform Art. 2014;29(3):125-35, https:// doi.org/10.21091/mppa.2014.3028.

64. Kenny DT, Driscoll T, Ackermann BJ. Is playing in the pit really the pits? Pain, strength, music performance anxiety, and workplace satisfaction in professional musicians in stage, pit, and combined stage/pit orchestras. Med Probl Perform Art. 2016;31(1):1-7, https://doi.org/10.21091/mppa.2016.1001.

65. Ackermann BJ, Kenny DT, O'Brien I, Driscoll TR. Sound practice - Improving occupational health and safety for professional orchestral musicians in Australia. Front Psychol. 2014;5:973, https://doi.org/10.3389/fpsyg.2014.00973.

66. Ackermann B, Driscoll T, Kenny DT. Musculoskeletal pain and injury in professional orchestral musicians in Australia. Med Probl Perform Art. 2012;27(4):181-7.

67. United Nations. World Economic Situation and Prospects 2016. United Nations; 2016.

68. Chan C, Driscoll T, Ackermann B. The usefulness of on-site physical therapy-led triage services for professional orchestral musicians - A national cohort study. BMC Musculoskelet Disord. 2013;14:98, https://doi.org/10.1186/1471-2474-14-98.

69. Allsop L, Ackland T. The prevalence of playing-related musculoskeletal disorders in relation to piano players' playing techniques and practising strategies. Music Perform Res. 2010;3(1):61-78. 
70. Rein S, Fabian T, Zwipp H, Heineck J, Weindel S. The influence of profession on functional ankle stability in musicians. Med Probl Perform Art. 2010;25(1):22-8.

71. Steinmetz A, Stang A, Kornhuber M, Röllinghoff M, Delank KS, Altenmüller E. From embouchure problems to embouchure dystonia? A survey of self-reported embouchure disorders in 585 professional orchestra brass players. Int Arch Occup Environ Health. 2014;87(7):783-92, https:// doi.org/10.1007/s00420-013-0923-4.

72. Pereira ÉF, Kothe F, Bleyer RTS, Teixeira CS. Workrelated stress and musculoskeletal complaints of orchestra musicians. Revista Dor 2014;15(2):112-6, https://doi. org/10.5935/1806-0013.20140025.

73. Heredia L, Hinkamp D, Brodsky M, Llapur C. Playingrelated problems among musicians of the Orquesta Buena Vista Social Club® and supporting bands. Med Probl Perform Art. 2014;29(2):80-5, https://doi.org/10.21091/mppa. 2014.2018.

74. De Queiroz JR, Mollica FB, Benetti P, de Araujo MA, Valera MC. Degree of chronic orofacial pain associated to the practice of musical instruments in orchestra's participants. Indian J Dent Res. 2014;25(1):28-31, https://doi. org/10.4103/0970-9290.131050.

75. Vaiano T, Guerrieri AC, Behlau M. Body pain in classical choral singers. Codas. 2013;25(4):303-9, https://doi. org/10.1590/S2317-17822013000400002.

76. Fotiadis DG, Fotiadou EG, Kokaridas DG, Mylonas AC. Prevalence of musculoskeletal disorders in professional symphony orchestra musicians in Greece: A pilot study concerning age, gender, and instrument-specific results. Med Probl Perform Art. 2013;28(2):91-5.

77. Teixeira ZLDO, Lã FBM, da Silva AG. Head and shoulder functional changes in flutists. Med Probl Perform Art. 2013;28(3):145-51.

78. Raymond DMr, Romeo JH, Kumke KV. A pilot study of occupational injury and illness experienced by classical musicians. Workplace Health Saf. 2012;60(1):19-24, https:// doi.org/10.3928/21650799-20111227-01.
79. Heikkilä J, Hamberg L, Meurman JH. Temporomandibular disorders: symptoms and facial pain in orchestra musicians in Finland. Music Med. 2012;4(3):171-6, https://doi. org/10.1177/1943862112445796.

80. Kaufman-Cohen Y, Ratzon NZ. Correlation between risk factors and musculoskeletal disorders among classical musicians. Occup Med (Lond). 2011;61(2):90-5, https://doi. org/10.1093/occmed/kqq196.

81. Barone Halleland H, Harris A, Sørnes S, Murison R, Ursin H. Subjective health complaints, stress, and coping in orchestra musicians. Med Probl Perform Art. 2009;24(2):58-62.

82. Yoshimura E, Fjellman-Wiklund A, Paul PM, Aerts C, Chesky K. Risk factors for playing-related pain among piano teachers. Med Probl Perform Art. 2008;23(3):107-13.

83. Nyman T, Wiktorin C, Mulder M, Johanson YL. Work postures and neck-shoulder pain among orchestra musicians. Am J Ind Med. 2007;50(5):370-6, https://doi.org/10.1002/ ajim.20454.

84. Nemoto K, Arino H. Hand and upper extremity problems in wind instrument players in military bands. Med Probl Perform Art. 2007;22(2):67-9.

85. Storms PR, Elkins CP, Strohecker EM. Embouchure dysfunction in Air Force Band brass musicians. Med Probl Perform Art. 2016;31(2):110-6, https://doi.org/10.21091/mppa. 2016.2019.

86. Berque P, Gray H, McFadyen A. Playing-related musculoskeletal problems among professional orchestra musicians in Scotland: A prevalence study using a validated instrument, the Musculoskeletal Pain Intensity and Interference Questionnaire for Musicians (MPIIQM). Med Probl Perform Art. 2016;31(2):78-86, https://doi.org/10.21091/mppa.2016.2015.

87. Sousa CM, Machado JP, Greten HJ, Coimbra D. Occupational diseases of professional orchestra musicians from Northern Portugal. A descriptive study. Med Probl Perform Art. 2016;31(1):8-12, https://doi.org/10.21091/mppa.2016.1002.

88. Mishra W, De A, Gangopadhyay S, Chandra AM. Playingrelated musculoskeletal disorders among Indian tabla players. Med Probl Perform Art. 2013;28(2):107-11. 
89. Knapik JJ, Jones SB, Darakjy S, Hauret KG, Nevin R, Grier T, et al. Injuries and injury risk factors among members of the United States Army Band. Am J Ind Med. 2007;50(12):951-61, https://doi.org/10.1002/ajim.20532.

90. Grier TL, Knapik JJ, Swedler D, Jones BH. Footwear in the United States Army Band: injury incidence and risk factors associated with foot pain. Foot (Edinb.). 2011;21(2):60-5, https://doi.org/10.1016/j.foot.2010.12.002.

91. Rickert D, Barrett M, Halaki M, Driscoll T, Ackermann B. A study of right shoulder injury in collegiate and professional orchestral cellists: An investigation using questionnaires and physical assessment. Med Probl Perform Art. 2012;27(2): $65-73$.

92. Lima RC, Pinheiro TM, Dias EC, de Andrade EQ. Development and prevention of work related disorders in a sample of Brazilian violinists. Work. 2015;51(2):273-80, https://doi. org/10.3233/WOR-141904.

93. Wahlström Edling C, Fjellman-Wiklund A. Musculoskeletal disorders and asymmetric playing postures of the upper extremity and back in music teachers: A pilot study. Med Probl Perform Art. 2009;24(3):113-8.

94. Barnes R, Attwood H, Blom J, Jankielsohn S, Smith T, Van Ede L, et al. Injury profile of musicians in the Bloemfonteinbased free state symphony orchestra: A short report. S Afr J Physiother. 2011;67(2):41-4, https://doi.org/10.4102/sajp. v67i2.45.

95. Leaver R, Harris EC, Palmer KT. Musculoskeletal pain in elite professional musicians from British symphony orchestras. Occup Med (Lond). 2011;61(8):549-55, https://doi. org/10.1093/occmed/kqr129.

96. Linari-Melfi M, Cantarero-Villanueva I, Fernández-Lao C, Fernández-de-las-Peñas C, Guisado-Barrilao R, ArroyoMorales M. Analysis of deep tissue hypersensitivity to pressure pain in professional pianists with insidious mechanical neck pain. BMC Musculoskelet Disord. 2011;12:268, https:// doi.org/10.1186/1471-2474-12-268.

97. Zamorano AM, Riquelme I, Kleber B, Altenmüller E, Hatem SM, Montoya P. Pain sensitivity and tactile spatial acuity are altered in healthy musicians as in chronic pain patients. Front Hum Neurosci. 2015;8:1016, https://doi.org/10.3389/ fnhum.2014.01016.

98. Khalsa SB, Shorter SM, Cope S, Wyshak G, Sklar E. Yoga ameliorates performance anxiety and mood disturbance in young professional musicians. Appl Psychophysiol Biofeedback. 2009;34(4):279-89, https://doi.org/10.1007/s10484-0099103-4.

99. Chan C, Driscoll T, Ackermann BJ. Effect of a musicians' exercise intervention on performance-related musculoskeletal disorders. Med Probl Perform Art. 2014;29(4):181-8, https://doi.org/10.21091/mppa.2014.4038.

100. Chan C, Driscoll T, Ackermann B. Exercise DVD effect on musculoskeletal disorders in professional orchestral musicians. Occup Med (Lond). 2014;64(1):23-30, https://doi. org/10.1093/occmed/kqt117.

101. Sakai N. Interosseous muscle pain in the pianist's hand: A description of 27 cases of "musician's hand". Med Probl Perform Art. 2007;22(1):24-5.

102. Wood GC. Prevalence, risk factors, and effects of performance-related medical disorders (PRMD) among tertiarytrained jazz pianists in Australia and the United States. Med Probl Perform Art. 2014;29(1):37-45, https://doi.org/ 10.21091/mppa.2014.1009.

103. Andersen LN, Roessler KK, Eichberg H. Pain among professional orchestral musicians: A case study in body culture and health psychology. Med Probl Perform Art. 2013;28(3):124-30.

104. Cupido C. Learning from experience: Exploring the wellbeing of professional opera singers. Muziki. 2016;13(2):80 107, https://doi.org/10.1080/18125980.2016.1182392.

105. Savvidou P, Willis B, Li M, Skubic M. Assessing injury risk in pianists: Using objective measures to promote selfawareness. MTNA e-journal. 2015;7(2):2-16.

106. Woldendorp KH, Boonstra AM, Tijsma A, Arendzen JH, Reneman MF. No association between posture and musculoskeletal complaints in a professional bassist sample. Eur J Pain. 2016;20(3):399-407, https://doi.org/10.1002/ejp.740. 
107. Papandreou M, Vervainioti A. Work-related musculoskeletal disorders among percussionists in Greece: a pilot study. Med Probl Perform Art. 2010;25(3):116-9.

108. Amorim MI, Jorge AIL. Association between temporomandiblar disorders and music performance anxiety in violinists. Occup Med (Lond). 2016;66(7):558-63, https://doi. org/10.1093/occmed/kqw080.

109. Ajidahun AT, Phillips J. Prevalence of musculoskeletal disorders among instrumental musicians at a Centre for Performing Arts in South Africa. Med Probl Perform Art. 2013;28(2):96-9.

110. Kuppens K, Struyf F, Nijs J, Cras P, Fransen E, Hermans L, et al. Exercise- and stress-induced hypoalgesia in musicians with and without shoulder pain: a randomized controlled crossover study. Pain Physician. 2016;19(2):59-68.

111. McCrary JM, Halaki M, Ackermann BJ. Effects of physical symptoms on music activity levels in skilled violinsts. Med Probl Perform Art. 2016;31(3):125-31, https://doi.org/ 10.21091/mppa.2016.3024.

112. Steinmetz A, Ridder PH, Methfessel G, Muche B. Professional musicians with craniomandibular dysfunctions treated with oral splints. Cranio. 2009;27(4):221-30, https://doi. org/10.1179/crn.2009.033.

113. Damian M, Zalpour C. Trigger point treatment with radial shock waves in musicians with nonspecific shoulder-neck pain: Data from a special physio outpatient clinic for musicians. Med Probl Perform Art. 2011;26(4):211-7.

114. Butler K, Winspur I. Retrospective case review of time taken for 130 professional musicians to fully return to playing their instruments following hand surgery. Hand Ther. 2009;14(3):69-74, https://doi.org/10.1258/ht.2009.009019.

115. Woldendorp KH, van de Werk P, Boonstra AM, Stewart RE, Otten E. Relation between muscle activation pattern and pain: An explorative study in a bassists population. Arch Phys Med Rehabil. 2013;94(6):1095-106, https://doi. org/10.1016/j.apmr.2012.11.036.

116. Nishiyama A, Tsuchida E. Relationship between wind instrument playing habits and symptoms of temporoman dibular disorders in non-professional musicians. Open Dent J. 2016;10:411-6, https://doi.org/10.2174/187421060 1610010411.

117. Moffit DM, Russ AC, Mansell JL. Marching band camp injury rates at the collegiate level. Med Probl Perform Art. 2015;30(2):96-9, https://doi.org/10.21091/mppa. 2015.2016.

118. Devroop K. Performance-related medical problems of university jazz studies majors. Muziki. 2016;13(1):91-9, https:// doi.org/10.1080/18125980.2016.1182384.

119. Rodríguez-Romero B, Pérez-Valiño C, Ageitos-Alonso B, Pértega-Díaz S. Prevalence and associated factors for musculoskeletal pain and disability among Spanish music conservatory. Med Probl Perform Art. 2016;31(4):193-200, https://doi.org/10.21091/mppa.2016.4035.

120. Savino E, Iannelli S, Forcella L, Narciso L, Faraso G, Bonifaci G, et al. Musculoskeletal disorders and occupational stress of violinists. J Biol Regul Homeost Agents. 2013;27(3):853-9.

121. Ackermann BJ, Kenny DT, Fortune J. Incidence of injury and attitudes to injury management in skilled flute players. Work. 2011;40(3):255-9, https://doi.org/10.3233/WOR2011-1227.

122. Jones Jr SB, Hernandez C. An investigation of the prevalence of upper limb neuropathies in different types of college musicians by use of neurometrix device. Int J Biol. 2010;2(1):132-42, https://doi.org/10.5539/ijb.v2n1p132.

123. Ginsborg J, Kreutz G, Thomas M, Williamon A. Healthy behaviours in music and non-music performance students. Health Edu. 2009;109(3):242-58, https://doi.org/10. 1108/09654280910955575.

124. Moore M, DeHaan L, Ehrenberg T, Gross L, Magembe C. Clinical assessment of shoulder impingement factors in violin and viola players. Med Probl Perform Art. 2008;23(4):155-63.

125. Kreutz G, Ginsborg J, Williamon A. Music students' health problems and health-promoting behaviours. Med Probl Perform Art. 2008;23(1):3-11. 
126. Steinmetz A, Möller H, Seidel W, Rigotti T. Playingrelated musculoskeletal disorders in music students-associated musculoskeletal signs. Eur J Phys Rehabil Med. 2012;48(4):625-33.

127. Árnason K, Árnason A, Briem K. Playing-related musculoskeletal disorders among Icelandic music students: differences between students playing classical vs rhythmic music. Med Probl Perform Art. 2014;29(2):74-9, https://doi. org/10.21091/mppa.2014.2017.

128. Stanhope J, Milanese S, Grimmer K. University woodwind students' experiences with playing-related injuries and their management: A pilot study. J Pain Res. 2014;7:133-48, https://doi.org/10.2147/JPR.S49620.

129. Ioannou CI, Altenmüller E. Approaches to and treatment strategies for playing-related pain problems among Czech instrumental music students: An epidemiological study. Med Probl Perform Art. 2015;30(3):135-42, https://doi. org/10.21091/mppa.2015.3027.

130. Hatheway M, Chesky K. Epidemiology of health concerns among collegiate student musicians participating in marching band. Med Probl Perform Art. 2013;28(4):242-51.

131. Lonsdale K, Boon OK. Playing-related health problems among instrumental music students at a university in Malaysia. Med Probl Perform Art. 2016;31(3):151-9, https:// doi.org/10.21091/mppa.2016.3028.

132. Beckett S, Seidelman L, Hanney WJ, Liu X, Rothschild CE. Prevalence of musculoskeletal injury among collegiate marching band and color guard members. Med Probl Perform Art. 2015;30(2):106-10, https://doi.org/10.21091/ mppa.2015.2018.

133. Baadjou VAE, Verbunt JAMCF, van Eijsden-Besseling MDF, Huysmans SMD, Smeets RJEM. The musician as (in)active athlete? Exploring the association between physical activity and musculoskeletal complaints in music students. Med Probl Perform Art. 2015;30(4):231-7, https://doi.org/10.21091/mppa.2015.4042.

134. Lamontagne V, Bélanger C. Pain-related and performance anxiety and their contribution to pain in music students:
A pilot study. Health Psychol Rep. 2015;3(1):59-68, https:// doi.org/10.5114/hpr.2015.47088.

135. Brandfonbrener A. History of playing-related pain in 330 university Freshman music students. Med Probl Perform Art. 2009;24(1):30-6.

136. Bruno S, Lorusso A, LAbbate N. Playing-related disabling musculoskeletal disorders in young and adult classical piano students. Int Arch Occup Environ Health. 2008;81(7):85560, https://doi.org/10.1007/s00420-007-0279-8.

137. Bragge P, Bialocerkowski A, McMeeken J. Musculoskeletal injuries in elite pianists: Prevalence and associated risk factors. Austr J Music Edu. 2008;1:18-31.

138. Barton R, Killian C, Bushee M, Callen J, Cupp T, Ochs B, et al. Occupational performance issues and predictors of dysfunction in college instrumentalists. Med Probl Perform Art. 2008;23(2):72-8.

139. Wanke EM, Kunath EK, Koch F, Davenport J, Weisser B, Groneberg DA, et al. Survey of health problems in musical theatre students: A pilot study. Med Probl Perform Art. 2012;27(4):205-11.

140. Daenen L, Roussel N, Cras P, Nijs J. Sensorimotor incongruence triggers sensory disturbances in professional violinists: An experimental study. Rheumatology. 2010;49(7): 1281-9, https://doi.org/10.1093/rheumatology/keq067.

141. Baskurt Z, Baskurt F, Kara B. Assessment of kinesthetic awareness and fine motor dexterity in music students with performance-related hand disorders. HealthMED. 2012;6(10):3395-402.

142. Wristen BW, Fountain SE. Relationships between depression, anxiety, and pain in a group of university music students. Med Probl Perform Art. 2013;28(3):152-8.

143. Martín López T, Farías Martínez J. Strategies to promote health and prevent musculoskeletal injuries in students from the High Conservatory of Music of Salamanca, Spain. Med Probl Perform Art. 2013;28(2):100-6.

144. Yoshimura E, Chesky K. The application of an ergonomically modified keyboard to reduce piano-related pain. MTNA e-journal. 2009;1(2):2-13. 
145. Lee SH, Carey S, Dubey R, Matz R. Intervention program in college instrumental musicians, with kinematics analysis of cello and flute playing: A combined program of yogic breathing and muscle strengthening-flexibility exercises. Med Probl Perform Art. 2012;27(2):85-94.

146. Laursen A, Chesky K. Addressing the NASM Health and Safety Standard through curricular changes in a brass methods course: An outcome study. Med Probl Perform Art. 2014;29(3):136-43, https://doi.org/10.21091/mppa. 2014.3029.

147. Barton R, Feinberg JR. Effectiveness of an educational program in health promotion and injury prevention for Freshman music majors. Med Probl Perform Art. 2008;23(2):47-53.

148. Kava KS, Larson CA, Stiller CH, Maher SF. Trunk endurance exercise and the effect on instrumental performance: A preliminary study comparing Pilates exericse and a trunk and proximal upper extremity endurance exercise program. Music Perform Res. 2010;3(1):1-30.

149. Piątkowska K, Wnuk B, Blicharska I, Rychlik M, Dumała J. Cervical pain in young professional musicians - Quality of life. Ortop Traumatol Rehabil. 2016;18(1):21-9, https:/doi. org/10.5604/15093492.1198832.

150. Ling CY, Loo FC, Hamedon TR. Knowledge of playingrelated musculoskeletal disorders among classical piano students at tertiary institutions in Malaysia. Med Probl Perform Art. 2016;31(4):201-4, https://doi.org/10.21091/ mppa.2016.4036.

151. Rickert DLL, Barrett MS, Ackermann BJ. Are music students fit to play? A case study of health awareness and injury attitudes amongst tertiary student cellists. Int J Music Educ. 2015;33(4):426-41, https://doi. org $/ 10.1177 / 0255761415582343$.

152. Park A, Guptill C, Sumsion T. Why music majors pursue music despite the risk of playing-related injuries. Med Probl Perform Art. 2007;22(3):89-96.

153. Kok LM, Haitjema S, Groenewegen KA, Rietveld AB. The influence of a sudden increase in playing time on playing-related musculoskeletal complaints in high-level amateur musicians in a longitudinal cohort study. PLoS One. 2016;11(9):e0163472, https://doi.org/10.1371/journal. pone. 0163472 .

154. Mehrparvar AH, Mostaghaci M, Gerami RF. Musculoskeletal disorders among Iranian instrumentalists. Med Probl Perform Art. 2012;27(4):193-6.

155. Wallace E, Klinge D, Chesky K. Musculoskeletal pain in trombonists. Results from the UNT Trombone Health Survey. Med Probl Perform Art. 2016;31(2):87-95, https://doi. org/10.21091/mppa.2016.2016.

156. Dhrithi MA, Agrawal PR, Aju K. Prevalence of playing-related musculoskeletal disorder (PRMSD) among amateur young guitar players. J Musculoskeletal Res. 2013;16(2):1330002, https://doi.org/10.1142/S02189577133 00020.

157. Ajidahun AT, Mudzi W, Myezwa H, Wood WA. Upper extremity disability among string instrumentalistsuse of the quick DASH and the NDI. Cogent Med. 2016;3:1234535, https://doi.org/10.1080/2331205X.2016.12 34535 .

158. Rohwer D. Health and wellness issues for adult band musicians. Med Probl Perform Art. 2008;23(2):54-8.

159. Levy JJ, Lounsbury JW. Big five personality traits and marching music injuries. Med Probl Perform Art. 2009; 24(3):135-40.

160. Sandell C, Frykman M, Chesky K, Fjellman-Wiklund A. Playing-related musculoskeletal disorders and stress-related health problems among percussionists. Med Probl Perform Art. 2009;24(4):175-80.

161. Ekholm O, Juel K, Bonde LO. Associations between daily musicking and health: Results from a nationwide survey in Denmark. Scand J Public Health 2016:44(7):726-32, https://doi.org/10.1177/1403494816664252.

162. Steinmetz A, Claus A, Hodges PW, Jull GA. Neck muscle function in violinists/violists with and without neck pain. Clin Rheumatol. 2016;35(4):1045-51, https://doi.org/10. 1007/s10067-015-3000-4. 
163. Steinmetz A, Jull GA. Sensory and sensorimotor features in violinists and violists with neck pain. Arch Phys Med Rehabil. 2013;94(12):2523-8, https://doi.org/10.1016/j.apmr. 2013.04.019.

164. Sakai N, Shimawaki S. Measurement of a number of indices of hand and movement angles in pianists with overuse disorders. J Hand Surg Eur. 2010;35(6):494-8, https://doi. org/10.1177/1753193409352405.

165. Moreno-Torres A, Rosset-Llobet J, Pujol J, Fàbregas S, Gonzalez-de-Suso JM. Work-related pain in extrinsic finger extensor musculature of instrumentalists is associated with intracellular $\mathrm{pH}$ compartmentation during exercise. PLoS One. 2010;5(2):e9091, https://doi.org/10.1371/journal. pone.0009091.

166. Steinmetz A, Seidel W, Muche B. Impairment of postural stabilization systems in musicians with playing-related musculoskeletal disorders. J Manipulative Physiol Ther. 2010;33(8):603-11, https://doi.org/10.1016/j.jmpt. 2010.08.006

167. Dawson WJ. Trauma to the high-level instrumentalist's hand and upper extremity: an epidemiologic and outcome study. Med Probl Perform Art. 2007;22(3):105-9.

168. Lahme A, Eibl I, Reichli FX. Typical musculoskeletal patterns in upper string players with neck and arm problems. Med Probl Perform Art. 2014;29(4):241-2, https://doi.org/ 10.21091/mppa.2014.4047.

169. Macfarlane JD, Rietveld ABM. A rheumatologist let loose in a performing arts clinic: The spectrum of musicians' complaints and their treatment. Med Probl Perform Art. 2009;24(4):185-7.

170. Yasuda E, Honda K, Hasegawa Y, Matsumura E, Fujiwara M, Hasegawa M, et al. Prevalence of temporomandibular disorders among junior high school students who play wind instruments Int J Occup Med Environ Health. 2016;29(1):69-76, https://doi.org/10.13075/ ijomeh.1896.00524.

171. Aparicio L, Lã FM, Silva AG. Pain and posture of children and adolescents who learn the accordion as compared with non-musician students. Med Probl Perform Art. 2016;31(4):187-92, https://doi.org/10.21091/mppa. 2016.4034

172. Vinci S, Smith A, Ranelli S. Selected physical characteristics and playing-related musculoskeletal problems in adolescent string instrumentalists. Med Probl Perform Art. 2015;30(3):143-51, https://doi.org/10.21091/mppa. 2015.3028

173. Russell JA, Benedetto RL. Perceived musculoskeletal discomfort among elementary, middle, and high school string players. J Res Music Educ. 2014;62(3):259-76, https://doi. org/10.1177/0022429414543307.

174. Kilanowski JF. Marching athletes: injuries and illnesses at band camp. MCN Am J Matern Child Nurs. 2008;33(6):33845, https://doi.org/10.1097/01.NMC.0000341252.95674.9f.

175. Ackermann B, Driscoll T. Attitudes and practices of parents of teenage musicians to health issues related to playing an instrument: A pilot study. Med Probl Perform Art. 2013;28(1):24-7.

176. Robitaille J, Guay M, Tousignant-Laflamme Y. Description of playing-related musculoskeletal pain in elite string students. Int Musculoskelet Med. 2015;37(3):125-31, https:// doi.org/10.1179/1753615415Y.0000000008.

177. McKechnie NC, Jacobs K. Physical and environmental factors contributing to music related injuries among children. Work. 2011;40(3):303-15, https://doi.org/10.3233/WOR-20111234.

178. Park KN, Kwon OY, Ha SM, Kim SJ, Choi HJ, Weon JH. Comparison of electromyographic activity and range of neck motion in violin students with and without neck pain during playing. Med Probl Perform Art. 2012;27(4):188-92.

179. Khalsa SBS, Butzer B, Shorter SM, Reinhardt KM, Cope S. Yoga reduces performance anxiety in adolescent musicians. Altern Ther Health Med. 2013;19(2):34-45.

180. Cooper SC, Hamann DL, Frost R. The effects of stretching exercises during rehearsals on string students' self-reported perceptions of discomfort. Update. 2012;30(2):71-6, https://doi.org/10.1177/8755123312438720. 
181. Saltychev M, Vastamäki H, Mattie R, McCormick Z, Vastamäki M, Laimi K. Psychometric properties of the Pain Numeric Rating Scale when applied to multiple body regions among professional musicians. PLoS One. 2016;11(9):e0161874, https://doi.org/10.1371/journal.pone.0161874.

182. Jang JY, Kwon JS, Lee DH, Bae JH, Kim ST. Clinical signs and subjective symptoms of temporomandibular disorders in instrumentalists. Yonsei Med J. 2016;57(6):1500-7, https://doi.org/10.3349/ymj.2016.57.6.1500.

183. Lonsdale K, Laakso EL, Tomlinson V. Contributing factors, prevention, and management of playing-related musculoskeletal disorders among flute players internationally. Med Probl Perform Art. 2014;29(3):155-62, https://doi. org/10.21091/mppa.2014.3032.

184. Artigues-Cano I, Bird HA. Hypermobility and proprioception in the finger joints of flautists. J Clin Rheumatol. 2014;20(4):203-8, https://doi.org/10.1097/RHU.000000000 0000109.

185. Akel S, Düger T. Psychosocial risk factors of musicians in Turkey: Use of the Job Content Questionnaire. Med Probl Perform Art. 2007;22(4):147-52.

186. Ajidahun AT, Phillips J. Content of a warm up programme for instrumental musicians: A Delphi study. Medicina Sportiva. 2013;9(2):2069-75.

187. Hoy D, Bain C, Williams G, March L, Brooks P, Blyth F, et al. A systematic review of the global prevalence of low back pain. Arthritis Rheum. 2012;64(6):2028-37, https:// doi.org/10.1002/art.34347.

188. Stanhope J. Physical performance and musculoskeletal disorders: are musicians and sportspeople on a level playing field? Perform Enhanc Health. 2016;4(1-2):18-26, https:// doi.org/10.1016/j.peh.2015.IJ.004.

189. National Association of Schools of Music [Internet]. Reston: National Association of Schools of Music; 2017 [cited 2018 Apr 10]. National Association of Schools of Music Handbook 2017-2018. Available from: https://nasm.artsaccredit.org/wp-content/uploads/sites/2/2017/12/M-Handbook-2017-2018.pdf.

190. Horvath J. Playing (less) hurt: An injury prevention guide for musicians. Milwaukee: Hal Leonard Books; 2010.

191. Australian Bureau of Statistics [Internet]. Canberra: The Bureau; 2011 [cited 2018 Apr 10]. Employment in Culture 6273.0 2011. Available from: http://www.ausstats.abs.gov. au/Ausstats/subscriber.nsf/0/24CE26B099300AF2CA257A DA00132646/\$File/62730_2011.pdf.

192. Henschke N, Kamper SJ, Maher CG. The epidemiology and economic consequences of pain. Mayo Clin Proc. 2015;90(1):139-47, https://doi.org/10.1016/j.mayocp. 2014.09.010.

193. Guptill C, Zaza C, Paul S. Treatment preferences of injured college student musicians. OTJR. 2005;25(1):4-8, https:// doi.org/10.1177/153944920502500102.

This work is available in Open Access model and licensed under a Creative Commons Attribution-NonCommercial 3.0 Poland License - http://creativecommons.org/ licenses/by-nc/3.0/pl/deed.en. 